\title{
Hopf Algebras, Renormalization and Noncommutative Geometry
}

\author{
Alain CONNES \\ IHES \\ Dirk KREIMER \\ Mainz Univ.
}

August 1998, IHES/M/98/60

\begin{abstract}
We explore the relation between the Hopf algebra associated to the renormalization of QFT and the Hopf algebra associated to the NCG computations of tranverse index theory for foliations.
\end{abstract}

\section{Introduction}

In [1] it was shown that the combinatorics of the subtraction procedure inherent to perturbative renormalization gives rise to a Hopf algebra $\mathcal{H}_{R}$ which provides a conceptual framework to understand the intricacies of the forest formula of Zimmermann.

In [2], it was shown that the delicate computational problem which arises from the transverse hypoelliptic theory of foliations, as formulated in noncommutative geometry, can only be settled thanks to a Hopf algebra $\mathcal{H}_{T}$ associated to each integer codimension. This Hopf algebra reduces transverse geometry, to a universal geometry of affine nature.

The aim of this paper is to establish a close relation between the above Hopf algebras $\mathcal{H}_{R}$ and $\mathcal{H}_{T}$. We shall first recall the first results of 20 which describe in the simplest case of codimension 1 the presentation of the Hopf algebra $\mathcal{H}_{T}$.

We shall then explain the origin of the Hopf algebra $\mathcal{H}_{R}$ from the renormalization of the divergences of QFT and show following [1] how $\mathcal{H}_{R}$ is used in concrete problems of renormalization theory. In the appendix we include the case of overlapping divergences.

We then give the presentation of the simplest model of $\mathcal{H}_{R}$ namely the Hopf algebra of rooted trees, and show that it is uniquely characterized as the solution of a universal problem in Hochschild cohomology. We then determine the formal Lie algebra $G$ such that $\mathcal{H}_{R}$ is obtained as the dual of the envelopping algebra of this Lie algebra. It turns out to be a refinement of the Lie algebra of formal vector fields in one dimension. We then show that many of the results 
of [2] actually extend to this refinement of formal vector fields. These results indicate that parallel to the ordinary differential calculus which underlies the transverse structure of foliations, the recipes of renormalization theory should be considered as a refined form of calculus and should be understandable on a conceptual ground.

Concretely, in the first section we show in Theorem (8) that the algebraic rules of the Hopf algebra $\mathcal{H}_{T}$ are the expression of the group law of composition of diffeomorphisms of $\mathbf{R}$ in terms of the coordinates $\delta_{n}$ given by the Taylor expansion of $-\log \left(\psi^{\prime}(x)\right)$ at $x=0$. In particular this shows that the antipode in $\mathcal{H}_{T}$ is, modulo a change of variables, the same as the operation of inversion of a formal power series for the composition law.

In the second section we begin by the simplest and most explicit examples of divergent integrals of the kind that are met in Quantum Field Theory computations.

We describe in this toy case the explicit counterterm construction and the immediate problem which arises from divergent subintegrations and explain how the Hopf algebra $\mathcal{H}_{R}$ finds the combinatorial solution of the subtraction problem from its antipode.

We next explain why the same holds in QFT (the treatment of overlapping divergences is postponed to the appendix).

In the third section we exhibit the precise relation and analogy between $\mathcal{H}_{R}$ and $\mathcal{H}_{T}$ to the point that the antipode in $\mathcal{H}_{R}$ appears as a direct analogue of the antipode in $\mathcal{H}_{T}$ which we understood above as the inversion of formal power series. The key nuance between the two Hopf algebras is that where $\mathcal{H}_{T}$ uses integers to label the Taylor expansion, the Hopf algebra $\mathcal{H}_{R}$ uses rooted trees for labels.

\section{The Hopf algebra $\mathcal{H}_{T}$}

The computation of the local index formula for transversally hypoelliptic operators ([2]) is governed by a very specific Hopf algebra $\mathcal{H}_{T}$, which only depends upon the codimension $n$ of the foliation. The structure of this Hopf algebra, its relation with the Lie algebra of formal vector fields as well as the computation of its cyclic cohomology have been done in [2].

In order to pursue the analogy between this development and the discovery by D. K. ([1]) of the Hopf algebra underlying renormalization, we shall recall here in all details the presentation and first properties of the Hopf algebra $\mathcal{H}_{T}$ in the simplest case of codimension one. Useless to say this does not dispense one from consulting [2], in particular in connection with the specific representation of $\mathcal{H}_{T}$ on crossed product algebras and the corresponding analysis.

We first define a bialgebra by generators and relations. As an algebra we view $\mathcal{H}_{T}$ as the envelopping algebra of the Lie algebra which is the linear span 
of $Y, X, \delta_{n}, n \geq 1$ with the relations,

$$
[Y, X]=X,\left[Y, \delta_{n}\right]=n \delta_{n},\left[\delta_{n}, \delta_{m}\right]=0 \forall n, m \geq 1,\left[X, \delta_{n}\right]=\delta_{n+1} \forall n \geq 1 .
$$

The coproduct $\Delta$ in $\mathcal{H}_{T}$ is defined by

$$
\Delta Y=Y \otimes 1+1 \otimes Y, \Delta X=X \otimes 1+1 \otimes X+\delta_{1} \otimes Y, \Delta \delta_{1}=\delta_{1} \otimes 1+1 \otimes \delta_{1}
$$

with $\Delta \delta_{n}$ defined by induction using (11) and the equality,

$$
\Delta\left(h_{1} h_{2}\right)=\Delta h_{1} \Delta h_{2} \quad \forall h_{j} \in \mathcal{H}_{T} .
$$

Lemma 1. The above presentation defines a Hopf algebra $\mathcal{H}_{T}$.

Proof. One checks that the Lie algebra relations (1) are fulfilled by the elements $\Delta(Y), \Delta(X), \Delta\left(\delta_{1}\right)$, so that, by the universal property of the envelopping algebra, $\Delta$ extends to an algebra homomorphism,

$$
\Delta: \mathcal{H}_{T} \rightarrow \mathcal{H}_{T} \otimes \mathcal{H}_{T}
$$

and using the uniqueness of the extension, one also checks the coassociativity.

One needs to show the existence of the antipode $S$. It is characterized abstractly as the inverse of the element $L(a)=a$ in the algebra of linear maps $L$ from $\mathcal{H}_{T}$ to $\mathcal{H}_{T}$ endowed with the product

$$
\left(L_{1} * L_{2}\right)(a)=\sum L_{1}\left(a_{(1)}\right) L_{2}\left(a_{(2)}\right) \quad \Delta a=\sum a_{(1)} \otimes a_{(2)}, a \in \mathcal{H}_{T} .
$$

A simple computation shows that $S$ is the unique antiautomorphism of $\mathcal{H}_{T}(n)$ such that,

$$
S(Y)=-Y \quad S\left(\delta_{1}\right)=-\delta_{1} \quad S(X)=-X+\delta_{1} Y .
$$

Note that the square of $S$ is not the identity.

In order to understand the Hopf algebra $\mathcal{H}_{T}$, we first analyse the commutative subalgebra generated by the $\delta_{n}$.

For each $n$ we let $\mathcal{H}_{n}$ be the subalgebra generated by $\delta_{1}, \ldots, \delta_{n}$,

$$
\mathcal{H}_{n}=\left\{P\left(\delta_{1}, \ldots, \delta_{n}\right) ; P \text { polynomial in } n \text { variables }\right\}
$$

We let $\mathcal{H}_{n, 0}$ be the ideal,

$$
\mathcal{H}_{n, 0}=\{P ; P(0)=0\} .
$$

By induction on $n$ one proves the following

Lemma 2. For each $n$ there exists $R_{n-1} \in \mathcal{H}_{n-1,0} \otimes \mathcal{H}_{n-1,0}$ such that $\Delta \delta_{n}=$ $\delta_{n} \otimes 1+1 \otimes \delta_{n}+R_{n-1}$. 
Proof. One has $\Delta \delta_{1}=\delta_{1} \otimes 1+1 \otimes \delta_{1}$, and a simple computation shows that,

$$
\Delta \delta_{2}=\delta_{2} \otimes 1+1 \otimes \delta_{2}+\delta_{1} \otimes \delta_{1}
$$

and,

$$
\Delta \delta_{3}=\delta_{3} \otimes 1+1 \otimes \delta_{3}+\delta_{1}^{2} \otimes \delta_{1}+\delta_{2} \otimes \delta_{1}+3 \delta_{1} \otimes \delta_{2} .
$$

In general, one determines $R_{n}$ by induction, using

$$
R_{n}=\left[X \otimes 1+1 \otimes X, R_{n-1}\right]+n \delta_{1} \otimes \delta_{n}+\left[\delta_{1} \otimes Y, R_{n-1}\right] .
$$

Since $\left[X, \mathcal{H}_{n-1,0}\right] \subset \mathcal{H}_{n, 0}$ and $\left[Y, \mathcal{H}_{n-1,0}\right] \subset \mathcal{H}_{n-1,0} \subset \mathcal{H}_{n, 0}$, one gets that $R_{n} \in \mathcal{H}_{n, 0} \otimes \mathcal{H}_{n, 0}$.

The equality (10) shows that $\mathcal{H}_{n}$ is not cocommutative for $n \geq 3$. However, since it is commutative, we shall determine the corresponding Lie algebra, using the Milnor-Moore theorem.

Let $\mathcal{A}_{n}^{1}$ be the Lie algebra of jets of order $(n+1)$ of vector fields on the line,

$$
f(x) \partial / \partial x \quad, \quad f(0)=f^{\prime}(0)=0
$$

modulo $x^{n+2} \partial / \partial x$.

Proposition 3. The Hopf algebra $\mathcal{H}_{n}$ is the dual of the envelopping agebra $\mathcal{U}\left(\mathcal{A}_{n}^{1}\right), \mathcal{H}_{n}=\mathcal{U}\left(\mathcal{A}_{n}^{1}\right)^{*}$.

Proof. For each $k \leq n$ we introduce a linear form $Z_{k, n}$ on $\mathcal{H}_{n}$

$$
\left\langle Z_{k, n}, P\right\rangle=\left(\frac{\partial}{\partial \delta_{k}} P\right)(0) .
$$

One has by construction,

$$
\left\langle Z_{k, n}, P Q\right\rangle=\left\langle Z_{k, n}, P\right\rangle Q(0)+P(0)\left\langle Z_{k, n}, Q\right\rangle .
$$

Note that $\varepsilon,\langle\varepsilon, P\rangle=P(0)$ is the counit of $\mathcal{H}_{n}$,

$$
\langle L \otimes \varepsilon, \Delta P\rangle=\langle\varepsilon \otimes L, \Delta P\rangle=\langle L, P\rangle \quad \forall P \in \mathcal{H}_{n} .
$$

(Check both sides on a monomial $P=\delta_{1}^{a_{1}} \ldots \delta_{n}^{a_{n}}$.)

Thus in the dual agebra $\mathcal{H}_{n}^{*}$ one can write (13) as

$$
\Delta Z_{k, n}=Z_{k, n} \otimes 1+1 \otimes Z_{k, n} .
$$

Moreover the $Z_{k, n}$ form a basis of the linear space of solutions of (15) and we just need to determine the Lie algebra structure determined by the bracket. Let,

$$
Z_{k, n}^{\prime}=(k+1) ! Z_{k, n}
$$


Let us show that $\left[Z_{k, n}^{\prime}, Z_{\ell, n}^{\prime}\right]=0$ if $k+\ell>n$, and that,

$$
\left[Z_{k, n}^{\prime}, Z_{\ell, n}^{\prime}\right]=(\ell-k) Z_{k+\ell, n}^{\prime}
$$

if $k+\ell \leq n$. Let $P=\delta_{1}^{a_{1}} \ldots \delta_{n}^{a_{n}}$ be a monomial. We need to compute $\left\langle\Delta P, Z_{k, n} \otimes Z_{\ell, n}-Z_{\ell, n} \otimes Z_{k, n}\right\rangle$. One has

$\Delta P=\left(\delta_{1} \otimes 1+1 \otimes \delta_{1}\right)^{a_{1}}\left(\delta_{2} \otimes 1+1 \otimes \delta_{2}+R_{1}\right)^{a_{2}} \ldots\left(\delta_{n} \otimes 1+1 \otimes \delta_{n}+R_{n-1}\right)^{a_{n}}$.

We look for the terms in $\delta_{k} \otimes \delta_{\ell}$ or $\delta_{\ell} \otimes \delta_{k}$ and take the difference. The latter is non zero only if all $a_{j}=0$ except $a_{q}=1$. Moreover since $R_{m}$ is homogeneous of degree $m+1$ one gets $q=k+\ell$ and in particular $\left[Z_{k, n}^{\prime}, Z_{\ell, n}^{\prime}\right]=0$ if $k+\ell>n$. One then computes by induction using (11) the bilinear part of $R_{m}$. One has $R_{1}^{(1)}=\delta_{1} \otimes \delta_{1}$, and from (11)

$$
R_{n}^{(1)}=\left[(X \otimes 1+1 \otimes X), R_{n-1}^{(1)}\right]+n \delta_{1} \otimes \delta_{n} .
$$

This gives

$$
R_{n-1}^{(1)}=\delta_{n-1} \otimes \delta_{1}+C_{n}^{1} \delta_{n-2} \otimes \delta_{2}+\ldots+C_{n}^{n-2} \delta_{1} \otimes \delta_{n-1} .
$$

Thus the coefficient of $\delta_{k} \otimes \delta_{\ell}$ is $C_{k+\ell}^{\ell-1}$ and we get

$$
\left[Z_{k, n}, Z_{\ell, n}\right]=\left(C_{k+\ell}^{\ell-1}-C_{k+\ell}^{k-1}\right) Z_{k+\ell, n} .
$$

One has $\frac{(k+1) !(\ell+1) !}{(k+\ell+1) !}\left(C_{k+\ell}^{\ell-1}-C_{k+\ell}^{k-1}\right)=\frac{\ell(\ell+1)-k(k+1)}{k+\ell+1}=\ell-k$ thus one gets (17). The elements $Z_{k, n}=\frac{x^{k+1}}{(k+1) !} \partial / \partial x$ of the Lie algebra $\mathcal{A}_{n}^{1}$ are related by (16) to $Z_{k, n}^{\prime}=x^{k+1} \partial / \partial x$ which satisfy the Lie algebra relations (17). The result then follows from the Milnor-Moore theorem.

The $\mathcal{A}_{n}^{1}$ form a projective system of Lie algebras, with limit the Lie algebra $\mathcal{A}^{1}$ of formal vector fields which vanish at order 2 at 0 . Thus the inductive limit $\mathcal{H}^{1}$ of the Hopf algebras $\mathcal{H}_{n}$ is,

$$
\mathcal{H}^{1}=\mathcal{U}\left(\mathcal{A}^{1}\right)^{*} .
$$

The Lie algebra $\mathcal{A}^{1}$ is a graded Lie algebra, with one parameter group of automorphisms,

$$
\alpha_{t}\left(Z_{n}\right)=e^{n t} Z_{n}
$$

which extends to $\mathcal{U}\left(\mathcal{A}^{1}\right)$ and transposes to $\mathcal{U}\left(\mathcal{A}^{1}\right)^{*}$ as

$$
\langle[Y, P], a\rangle=\left\langle P, \frac{\partial}{\partial t} \alpha_{t}(a)_{t=0}\right\rangle \quad \forall P \in \mathcal{H}^{1}, a \in \mathcal{U}\left(\mathcal{A}^{1}\right) .
$$

Indeed $\left(\alpha_{t}\right)^{t}$ is a one parameter group of automorphisms of $\mathcal{H}^{1}$ such that

$$
\alpha_{t}^{t}\left(\delta_{n}\right)=e^{n t} \delta_{n} .
$$


Now, using the Poincaré-Birkhoff-Witt theorem, we take the basis of $\mathcal{U}\left(\mathcal{A}^{1}\right)$ given by the monomials,

$$
Z_{n}^{a_{n}} Z_{n-1}^{a_{n-1}} \ldots Z_{2}^{a_{2}} Z_{1}^{a_{1}}, a_{j} \geq 0 .
$$

To each $L \in \mathcal{U}\left(\mathcal{A}^{1}\right)^{*}$ one associates the formal power series

$$
\sum \frac{L\left(Z_{n}^{a_{n}} \ldots Z_{1}^{a_{1}}\right)}{a_{n} ! \ldots a_{1} !} x_{1}^{a_{1}} \ldots x_{n}^{a_{n}},
$$

in the commuting variables $x_{j}, j \in \mathbb{N}$.

It follows from [3] 2.7.5 that we obtain in this way an isomorphism of the algebra of polynomials $P\left(\delta_{1}, \ldots, \delta_{n}\right)$ on the algebra of polynomials in the $x_{j}$ 's. To determine the formula for $\delta_{n}$ in terms of the $x_{j}$ 's, we just need to compute

$$
\left\langle\delta_{n}, Z_{n}^{a_{n}} \ldots Z_{1}^{a_{1}}\right\rangle .
$$

Note that, by homogeneity, (27) vanishes unless $\sum j a_{j}=n$.

For $n=1$, we get

$$
\rho\left(\delta_{1}\right)=x_{1}
$$

where $\rho$ is the above isomorphism.

We determine $\rho\left(\delta_{n}\right)$ by induction, using the derivation

$$
D(P)=\sum \delta_{n+1} \frac{\partial}{\partial \delta_{n}}(P)
$$

(which corresponds to $P \rightarrow[X, P]$ ).

One has by construction,

$$
\left\langle\delta_{n}, a\right\rangle=\left\langle\delta_{n-1}, D^{t}(a)\right\rangle \quad \forall a \in \mathcal{U}\left(\mathcal{A}^{1}\right)
$$

where $D^{t}$ is the transpose of $D$.

By definition of $Z_{n}$ as a linear form (12) one has,

$$
D^{t} Z_{n}=Z_{n-1}, n \geq 2, D^{t} Z_{1}=0 .
$$

Moreover the compatibility of $D^{t}$ with the coproduct of $\mathcal{H}^{1}$ is given by

$$
D^{t}(a b)=D^{t}(a) b+a D^{t}(b)+\left(\delta_{1} a\right) \partial_{t} b \quad \forall a, b \in \mathcal{U}\left(\mathcal{A}^{1}\right)
$$

where $a \rightarrow \delta_{1} a$ is the natural action of the algebra $\mathcal{H}^{1}$ on its dual

$$
\left\langle P, \delta_{1} a\right\rangle=\left\langle P \delta_{1}, a\right\rangle \quad \forall P \in \mathcal{H}^{1}, a \in \mathcal{U}\left(\mathcal{A}^{1}\right) .
$$

Lemma 4. When restricted to $\mathcal{U}\left(\mathcal{A}^{2}\right), D^{t}$ is the unique derivation, with values in $\mathcal{U}\left(\mathcal{A}^{1}\right)$ satisfying (3马), moreover

$$
D^{t}\left(Z_{n}^{a_{n}} \ldots Z_{2}^{a_{2}} Z_{1}^{a_{1}}\right)=D^{t}\left(Z_{n}^{a_{n}} \ldots Z_{2}^{a_{2}}\right) Z_{1}^{a_{1}}+Z_{n}^{a_{n}} \ldots Z_{2}^{a_{2}} \frac{a_{1}\left(a_{1}-1\right)}{2} Z_{1}^{a_{1}-1} .
$$


Proof. The equality $\Delta \delta_{1}=\delta_{1} \otimes 1+1 \otimes \delta_{1}$ shows that $a \rightarrow \delta_{1} a$ is a derivation of $\mathcal{U}\left(\mathcal{A}^{1}\right)$. One has $\delta_{1} Z_{n}=0$ for $n \neq 1$ so that $\delta_{1}=0$ on $\mathcal{U}\left(\mathcal{A}^{2}\right)$ and the first statement follows from (31) and (32). The second statement follows from,

$$
D^{t}\left(Z_{1}^{m}\right)=\frac{m(m-1)}{2} Z_{1}^{m-1}
$$

which one proves by induction on $m$ using (32).

Motivated by the first part of the lemma, we enlarge the Lie algebra $\mathcal{A}^{1}$ by adjoining an element $Z_{-1}$ such that,

$$
\left[Z_{-1}, Z_{n}\right]=Z_{n-1} \quad \forall n \geq 2,
$$

we then define $Z_{0}$ by

$$
\left[Z_{-1}, Z_{1}\right]=Z_{0},\left[Z_{0}, Z_{k}\right]=k Z_{k} .
$$

The obtained Lie algebra $\mathcal{A}$, is the Lie algebra of formal vector fields with $Z_{0}=x \frac{\partial}{\partial x}, Z_{-1}=\frac{\partial}{\partial x}$ and as above $Z_{n}=\frac{x^{n+1}}{(n+1) !} \frac{\partial}{\partial x}$.

Let $\mathcal{L}$ be the left ideal in $\mathcal{U}(\mathcal{A})$ generated by $Z_{-1}, Z_{0}$,

Proposition 5. The linear map $D^{t}: \mathcal{U}\left(\mathcal{A}^{1}\right) \rightarrow \mathcal{U}\left(\mathcal{A}^{1}\right)$ is uniquely determined by the equality $D^{t}(a)=\left[Z_{-1}, a\right] \bmod \mathcal{L}$.

Proof. Let us compare $D^{t}$ with the bracket with $Z_{-1}$. By Lemma 4 , they agree on $\mathcal{U}\left(\mathcal{A}^{2}\right)$. Let us compute $\left[Z_{-1}, Z_{1}^{m}\right]$. One has

$$
\left[Z_{-1}, Z_{1}^{m}\right]=\frac{m(m-1)}{2} Z_{1}^{m-1}+m Z_{1}^{m-1} Z_{0} .
$$

For each monomial $Z_{n}^{a_{n}} \ldots Z_{1}^{a_{1}}$ one has $D^{t}(a)-\left[Z_{-1}, a\right] \in \mathcal{L}$. Thus this holds for any $a \in \mathcal{U}\left(\mathcal{A}^{1}\right)$. Moreover, using the basis of $\mathcal{U}(\mathcal{A})$ given by the

$$
Z_{n}^{a_{n}} \ldots Z_{1}^{a_{1}} Z_{0}^{a_{0}} Z_{-1}^{a_{-1}}
$$

we see that $\mathcal{U}(\mathcal{A})$ is the direct sum $\mathcal{L} \oplus \mathcal{U}\left(\mathcal{A}^{1}\right)$.

We now define a linear form $L_{0}$ on $\mathcal{U}(\mathcal{A})$ by

$$
L_{0}\left(Z_{n}^{a_{n}} \ldots Z_{1}^{a_{1}} Z_{0}^{a_{0}} Z_{-1}^{a_{-1}}\right)=0 \text { unless } a_{0}=1, a_{j}=0 \quad \forall j,
$$

and $L_{0}\left(Z_{0}\right)=1$.

Lemma 6. For any $n \geq 1$ one has

$$
\left\langle\delta_{n}, a\right\rangle=L_{0}\left([\underbrace{\ldots}_{n \text { times }}\left[Z_{-1}, a\right] \ldots]\right) \quad \forall a \in \mathcal{U}\left(\mathcal{A}^{1}\right) .
$$

Proof. Let us first check it for $n=1$. We let $a=Z_{n}^{a_{n}} \ldots Z_{1}^{a_{1}}$. Then the degree of $a$ is $\sum j a_{j}$ and $L_{0}\left(\left[Z_{-1}, a\right]\right) \neq 0$ requires $\sum j a_{j}=1$ so that the 
only possibility is $a_{1}=1, a_{j}=0 \quad \forall j$. In this case one gets $L_{0}\left(\left[Z_{-1}, Z_{1}\right]\right)=$ $L_{0}\left(Z_{0}\right)=1$. Thus by (28) we get the equality of Lemma 6 for $n=1$.

For the general case note first that $\mathcal{L}$ is stable under right multiplication by $Z_{-1}$ and hence by the derivation $\left[Z_{-1}, \cdot\right]$. Thus one has

$$
\left(D^{t}\right)^{n}(a)=\left[Z_{-1}, \ldots\left[Z_{-1}, a\right] \ldots\right] \bmod \mathcal{L} \quad \forall a \in \mathcal{U}\left(\mathcal{A}^{1}\right) .
$$

Now for $a \in \mathcal{L}$ one has $L_{0}\left(\left[Z_{-1}, a\right]\right)=0$. Indeed writing

$$
a=\left(Z_{n}^{a_{n}} \ldots Z_{1}^{a_{1}}\right)\left(Z_{0}^{a_{0}} Z_{-1}^{a_{-1}}\right)=b c
$$

with $b \in \mathcal{U}\left(\mathcal{A}^{1}\right), c=Z_{0}^{a_{0}} Z_{-1}^{a_{-1}}$, one has $\left[Z_{-1}, a\right]=\left[Z_{-1}, b\right] c+b\left[Z_{-1}, c\right]$.

Since $b \in \mathcal{U}\left(\mathcal{A}^{1}\right)$ and $\left[Z_{-1}, c\right]$ has strictly negative degree one has $L_{0}\left(b\left[Z_{-1}, c\right]\right)$ $=0$. Let $Z_{n}^{b_{n}} \ldots Z_{1}^{b_{1}} Z_{0}^{b_{0}}$ be a non zero component of $\left[Z_{-1}, b\right]$, then unless all $b_{i}$ are 0 it contributes by 0 to $L_{0}\left(\left[Z_{-1}, b\right] c\right)$. But $\left[Z_{-1}, b\right] \in \mathcal{U}\left(\mathcal{A}^{0}\right)_{0}$ has no constant term. Thus one has

$$
L_{0}\left(\left[Z_{-1}, a\right]\right)=0 \quad \forall a=Z_{n}^{a_{n}} \ldots Z_{1}^{a_{1}} Z_{0}^{a_{0}} Z_{-1}^{a_{-1}}
$$

except if all $a_{j}=0, j \neq 1$ and $a_{1}=1 . L_{0}\left(\left[Z_{-1}, Z_{1}\right]\right)=1$.

Using (31) one has $\left\langle\delta_{n}, a\right\rangle=\left\langle\delta_{1},\left(D^{t}\right)^{n-1}(a)\right\rangle$ and the lemma follows.

One can now easily compute the first values of $\rho\left(\delta_{n}\right), \rho\left(\delta_{1}\right)=x_{1}, \rho\left(\delta_{2}\right)=$ $x_{2}+\frac{x_{1}^{2}}{2}, \rho\left(\delta_{3}\right)=x_{3}+x_{2} x_{1}+\frac{x_{1}^{3}}{2}, \rho\left(\delta_{4}\right)=x_{4}+x_{3} x_{1}+2 x_{2}^{2}+2 x_{2} x_{1}^{2}+\frac{3}{4} x_{1}^{4}$.

The affine structure provided by the $\delta_{n}$ has the following compatibility with left multiplication in $\mathcal{U}\left(\mathcal{A}^{1}\right)$.

Lemma 7. a) One has $R_{n-1}=\sum R_{n-1}^{k} \otimes \delta_{k}, R_{n-1}^{k} \in \mathcal{H}_{n-1,0}$.

b) For fixed $a_{0} \in \mathcal{U}\left(\mathcal{A}^{1}\right)$ there are $\lambda_{n}^{k} \in \mathbb{C}$ such that

$$
\left\langle\delta_{n},\left(a_{0} a\right)\right\rangle=\left\langle\delta_{n}, a_{0}\right\rangle \varepsilon(a)+\sum \lambda_{n}^{k}\left\langle\delta_{k}, a\right\rangle .
$$

Proof. a) By induction using (7). b) Follows, using $\lambda_{n}^{k}=\left\langle R_{n-1}^{k}, a_{0}\right\rangle$.

The antipode $S$ in $\mathcal{U}\left(\mathcal{A}^{1}\right)$ is the unique antiautomorphism such that

$$
S Z_{n}=-Z_{n} \quad \forall n .
$$

It is non trivial to express in terms of the coordinates $\delta_{n}$.

In fact if we use the basis $Z_{j}$ of $\mathcal{A}^{1}$ but in reverse order to construct the map $\rho$ we obtain a map $\widetilde{\rho}$ whose first values are $\widetilde{\rho}\left(\delta_{1}\right)=z_{1}, \widetilde{\rho}\left(\delta_{2}\right)=z_{2}+\frac{z_{1}^{2}}{2}$, $\widetilde{\rho}\left(\delta_{3}\right)=z_{3}+3 z_{1} z_{2}+\frac{1}{2} z_{1}^{3}, \widetilde{\rho}\left(\delta_{4}\right)=z_{4}+2 z_{2}^{2}+6 z_{1} z_{3}+9 z_{1}^{2} z_{2}+\frac{3}{4} z_{1}^{4}$.

One has

$$
\left\langle\delta_{n}, S\left(Z_{m}^{a_{m}} \ldots Z_{1}^{a_{1}}\right)\right\rangle=(-1)^{\sum a_{j}}\left\langle\delta_{n}, Z_{1}^{a_{1}} \ldots Z_{m}^{a_{m}}\right\rangle
$$

so that

$$
\rho\left(S^{t} \delta_{n}\right)=\sum\left\langle\delta_{n}, S\left(Z_{m}^{a_{m}} \ldots Z_{1}^{a_{1}}\right)\right\rangle x_{1}^{a_{1}} \ldots x_{m}^{a_{m}}=
$$




$$
=\sum(-1)^{\sum a_{j}}\left\langle\delta_{n}, Z_{1}^{a_{1}} \ldots Z_{m}^{a_{m}}\right\rangle x_{1}^{a_{1}} \ldots x_{m}^{a_{m}}=\widetilde{\rho}\left(\delta_{n}\right)
$$

with $z_{j}=-x_{j}$ in the latter expression.

Thus $\rho\left(S^{t} \delta_{1}\right)=-x_{1}, \rho\left(S^{t} \delta_{2}\right)=-x_{2}+\frac{x_{1}^{2}}{2}, \rho\left(S^{t} \delta_{3}\right)=-x_{3}+3 x_{1} x_{2}-\frac{x_{1}^{3}}{2}$, $\rho\left(S^{t} \delta_{4}\right)=-x_{4}+2 x_{2}^{2}+6 x_{1} x_{3}-9 x_{1}^{2} x_{2}+\frac{3}{4} x_{1}^{4}$. We thus get

$$
S^{t} \delta_{1}=-\delta_{1}, S^{t} \delta_{2}=-\delta_{2}+\delta_{1}^{2}, S^{t} \delta_{3}=-\delta_{3}+4 \delta_{1} \delta_{2}-2 \delta_{1}^{3}, \ldots
$$

The meaning of all the above computations and their relation to the standard calculus of Taylor expansions is clarified by the following theorem ([2]).

Theorem 8. Let $G_{2}$ be the group of formal diffeomorphisms of $\mathbb{R}$, of the form $\psi(x)=x+o(x)$. For each $n$, let $\gamma_{n}$ be the functional on $G_{2}$ defined by,

$$
\gamma_{n}\left(\psi^{-1}\right)=\left(\partial_{x}^{n} \log \psi^{\prime}(x)\right)_{x=0} .
$$

The equality $\Theta\left(\delta_{n}\right)=\gamma_{n}$ determines a canonical isomorphism $\Theta$ of the Hopf algebra $\mathcal{H}^{1}$ with the Hopf algebra of coordinates on the group $G_{2}$.

We refer to Theorem 8 of [2] for the proof, as well as for the more elaborate structure of the Hopf algebra $\mathcal{H}_{T}$. This theorem certainly shows that the antipode, i.e. the map $\psi \rightarrow \psi^{-1}$ is certainly non trivial to compute. Note also that the expression $\sigma=\delta_{2}-\frac{1}{2} \delta_{1}^{2}$ is uniquely characterized by

$$
\rho(\sigma)=x_{2}
$$

which suggests to define higher analogues of the Schwartzian as $\rho^{-1}\left(x_{n}\right)$.

\section{The physics of renormalization and the Hopf algebra of rooted trees}

In this section we want to motivate the Hopf algebra structure behind the process of renormalization in Quantum Field Theories (QFTs) [1] and show how relations to the Hopf algebra of the previous section emerge.

The renormalization procedure appears as the cure for the disease caused by the unavoidable presence of UV divergences in QFTs which describe the physics of local quantized fields. Such QFTs describe successfully all known particle physics phenomenology.

The point of departure of the renormalization procedure is to alter the original Lagrangian by an infinite series of counterterms labelled by Feynman graphs, whose sole purpose is to cancel the UV-divergences coming from the presence of ill-defined integrals in the perturbative expansion of the theory.

Recall that the perturbative expansion of the functional integral appears as a sum labelled by Feynman graphs $\Gamma$. To each of these graphs corresponds an integral $I_{\Gamma}$ which is in general ill-defined. To compensate for the resulting 
infinities one adds to the original Lagrangian $L_{0}$ which appears as the argument of the exponential, an infinite series of counterterms $\sum_{\Gamma} L_{\Gamma}$, each term in the series corresponding to a Feynman graph $\Gamma$. The difficulty in finding the cutoff dependent counterterm Lagrangian $\sum L_{\Gamma}$ comes only from the presence of ill-defined subintegrations (usually dubbed subdivergences) in the integral $I_{\Gamma}$. Indeed in the special case of a diagram without subdivergences the counterterm is simply (in the MS scheme) just the pole part of $I_{\Gamma}$.

As soon as subdivergences are present the extraction of $L_{\Gamma}$ is much more complicated since we want to take into account the previous subtractions which is necessary to maintain locality in the theory.

This obviously generates complicated combinatorial problems, which for the first time, acquire mathematical meaning thanks to the Hopf algebra $\mathcal{H}_{R}$.

\section{A Toy Model}

It is possible to study the basic properties of the renormalization procedure with the help of toy models, to which we now turn.

In the following we will consider integrals of the form

$$
x(c):=\int_{0}^{\infty} \frac{1}{y+c} d y
$$

for $c>0$, which are to be regarded as functions of the parameter $c$.

As it stands such an integral is ill-defined, due to its divergence at the upper boundary. Power counting reveals the presence of a logarithmic singularity, and in this respect the integral behaves no better or worse than a logarithmic divergent integral in QFT, which one typically confronts due to the presence of UV divergences in loop integrations.

We will introduce a regularization,

$$
x(c)=\int_{0}^{\infty} y^{-\epsilon} \frac{1}{y+c} d y,
$$

where $\epsilon$ is a small positive parameter.

We now easily evaluate the above integral

$$
x(c)=B(\epsilon, 1-\epsilon) c^{-\epsilon},
$$

where the presence of the pole term $\sim \Gamma(\epsilon)=\Gamma(1+\epsilon) / \epsilon$ indicates the $U V$ divergence in the integral 1 .

The process of renormalization demands the subtraction of this UV divergence, and at this level we can straightforwardly proceed by a simple subtraction

$$
\begin{gathered}
x(c)-x(1)=\int_{0}^{\infty} y^{-\epsilon} \frac{(1-c)}{(y+c)(y+1)} d y=B(\epsilon, 1-\epsilon)\left(c^{-\epsilon}-1\right) \\
{ }^{1} B(\epsilon, 1-\epsilon)=\Gamma(1+\epsilon) \Gamma(1-\epsilon) / \epsilon, \Gamma(1+x)=\exp (-\gamma x) \exp \left(\sum_{j=2}^{\infty} \zeta(j) x^{j} / j\right),|x|<1 .
\end{gathered}
$$


which is evidently finite if we send $\epsilon \rightarrow 0$.

Here, $-x(1)$ acts as the counterterm for the ill-defined function $x(c)$, and the difference $x(c)-x(1)$ corresponds to the renormalized function associated to $x(c)$.

Physicists have good reason to demand that a counterterm like $-x(1)$ above is independent of the external parameter $c$, as to maintain locality in the theory. Before we explain this in more detail we want to generalize this simple example to the presence of subdivergences.

We consider

$$
\begin{aligned}
x_{2}(c) & :=\int_{0}^{\infty} \int_{0}^{\infty} y_{1}^{-\epsilon} y_{2}^{-\epsilon} \frac{1}{y_{1}+c} \frac{1}{y_{2}+y_{1}} d y_{1} d y_{2} \\
& =\int_{0}^{\infty} y_{1}^{-\epsilon} \frac{1}{y_{1}+c} x\left(y_{1}\right) d y_{1} .
\end{aligned}
$$

We say that $x_{2}(c)$ has the function $x\left(y_{1}\right)$ as a subdivergence, but it still is overall divergent itself.

Powercounting reveals that there is a divergent sector when the $y_{2}$ integration variable tends to infinity for any fixed $y_{1}$, and when $y_{1}, y_{2}$ tend to infinity jointly. There are no divergences when $y_{2}$ is kept fixed and $y_{1}$ tends to infinity, though. All the divergences are of logarithmic nature.

Having successfully eliminated the divergence in the previous example by a naive subtraction procedure, it is interesting to see if we can eliminate the divergences in $x_{2}(c)$ by subtracting $x_{2}(1)$ :

$$
\begin{aligned}
x_{2}(c)-x_{2}(1) & =\int_{0}^{\infty} y_{1}^{-\epsilon} y_{2}^{-\epsilon} \frac{(1-c)}{\left(y_{1}+c\right)\left(y_{1}+1\right)} \frac{1}{y_{2}+y_{1}} d y_{1} d y_{2} \\
& =B(\epsilon, 1-\epsilon) \int_{0}^{\infty} y_{1}^{-2 \epsilon} \frac{(1-c)}{\left(y_{1}+c\right)\left(y_{1}+1\right)} d y_{1} \\
& =B(\epsilon, 1-\epsilon)\left[B(2 \epsilon, 1-2 \epsilon) c^{-2 \epsilon}-B(2 \epsilon, 1-2 \epsilon)\right] \\
& =-\frac{\log (c)}{\epsilon}+\text { finite terms. }
\end{aligned}
$$

Unfortunately, this expression still suffers from a divergence in the $y_{2}$ integration, and we were thus not successful with this naive attempt.

Actually, we find that the divergence is $\sim \log (c)$. The parameter $c$ in our toy model is the remaining scale of the Green function. In realistic QFTs, this scale is furnished typically by an external momentum $q$, say, and divergences of the form $\log \left(q^{2}\right) / \epsilon$ are non-local divergences: upon Fourier-transformation, they involve the logarithm of a differential operator, for example the logarithm of an external $q^{2}$ would translate as $\log (\square)$. Such terms can not be absorbed by local counterterms, and are strictly to be avoided if one wants to remain in the context of a local field theory. In the context of field theory, locality restricts counterterms to be polynomial in momenta. 
Correspondingly, in the context of our toy model, we thus look for counterterms which are at most polynomial in the parameter $c$.

The failure above was twofold: the naive subtraction $-x_{2}(1)$ not only failed to render $x_{2}(c)$ finite, but also this failure could only be absorbed by a non-local counterterm $\sim \log (c) / \epsilon$. To find a local counterterm, some more work is needed.

Following the guidance of field theory we associate to $x_{2}(c)$ (corresponding to a bare Green function) a function which has its subdivergences subtracted (a transition in field theory achieved by the $\bar{R}$ operation):

$$
\begin{aligned}
\overline{x_{2}}(c) & :=x_{2}(c)-x(c) x(1) \equiv \int_{0}^{\infty} y_{1}^{-\epsilon} y_{2}^{-\epsilon} \frac{1}{y_{1}+c}\left(\frac{1}{y_{2}+y_{1}}-\frac{1}{y_{2}+1}\right) d y_{1} d y_{2} \\
& =B(\epsilon, 1-\epsilon)\left[B(2 \epsilon, 1-2 \epsilon) c^{-2 \epsilon}-B(\epsilon, 1-\epsilon) c^{-\epsilon}\right] .
\end{aligned}
$$

Note that the subtraction term $-x(c) x(1)$ involves the counterterm $-x(1)$ times the analytic expression, $x(c)$, which we obtain from $x_{2}(c)$ when we set the subdivergence $x\left(y_{1}\right)$ in $x_{2}(c)$ to one.

We realize that $x_{2}^{R}(c)=\lim _{\epsilon \rightarrow 0}\left[\overline{x_{2}}(c)-\overline{x_{2}}(1)\right]$ is a well-defined finite expression, the finite renormalized Green function $x_{2}^{R}(c)$,

$$
\begin{aligned}
x_{2}^{R}(c)= & \lim _{\epsilon \rightarrow 0}\left\{B(\epsilon, 1-\epsilon)\left[B(2 \epsilon, 1-2 \epsilon) c^{-2 \epsilon}-B(\epsilon, 1-\epsilon) c^{-\epsilon}\right]\right. \\
& -B(\epsilon, 1-\epsilon)[B(2 \epsilon, 1-2 \epsilon)-B(\epsilon, 1-\epsilon)]\} \\
= & \frac{1}{2} \log ^{2}(c)
\end{aligned}
$$

and thus identify

$$
-\overline{x_{2}}(1)=-B(\epsilon, 1-\epsilon)[B(2 \epsilon, 1-2 \epsilon)-B(\epsilon, 1-\epsilon)]
$$

with the counterterm associated to $x_{2}(c)$.

Note that $x_{2}^{R}(1)=0$, by construction. The renormalized Green function $x_{2}^{R}(c)$ becomes a power series in $\log (c)$ (without constant term). Note further that we can write an integral representation for it which eliminates the necessity to introduce a regularization at all:

$$
x_{2}^{R}(c)=\int_{0}^{\infty} \int_{0}^{\infty}\left[\frac{1}{x+c}\left[\frac{1}{y+x}-\frac{1}{y+1}\right]-\frac{1}{x+1}\left[\frac{1}{y+x}-\frac{1}{y+1}\right]\right] d y d x .
$$

This could be directly obtained following the BPHZ approach, and what we have just seen is the equivalence between on-shell renormalization (subtraction at the on-shell value $c=1$ ) and the BPHZ renormalization in the toy model.

The above example shows how to find a local counterterm for an ill-defined integral with ill-defined subintegrations. We first eliminated the ill-defined subintegration by a counterterm, and then proceeded to construct the counterterm for the integral as a whole. In QFT one proceeds in the same manner. A 
bare Green-function, given by an ill-defined integral, will suffer from a plethora of ill-defined subintegrations in general.

These subintegrations can be disjoint, nested or overlapping [4]. We will see later that the overlapping case resolves into the other ones. This result was effectively already obtained in [1, 5, 6], and also known to others. An example how to resolve overlapping divergences in the case of $\phi^{3}$ theory in six dimensions will be given in an appendix.

Thus, we introduce at this stage a generalization of the above toy model allowing only for arbitrary nested or disjoint subdivergences.

This motivates to generalize the example to functions $x_{t}(c)$ of an external parameter $c$, indexed by a rooted tree $t$, due to the fact that any configurations of nested or disjoint subdivergences can be described by a rooted tree. The formal definition of a rooted tree is postponed to the next section, while here we continue to gain experience in the treatment of functions having nested and disjoint subdivergences.

We define for a tree $t$ with $m$ vertices, enumerated such that the root has number 1 ,

$$
x_{t}(c):=\int_{0}^{\infty} \frac{1}{y_{1}+c} \prod_{i=2}^{m} \frac{1}{y_{i}+y_{j(i)}} y_{m}^{-\epsilon} d y_{m} \ldots y_{1}^{-\epsilon} d y_{1}, \forall c>0,
$$

where $j(i)$ is the number of the vertex to which the $i$-th vertex is connected via its incoming edge.

We can write this as

$$
x_{t}(c):=\int \frac{1}{y+c} \prod_{j=1}^{r} x_{t_{j}}(y) y^{-\epsilon} d y,
$$

if the root of $t$ connects to $r$ trees $t_{j}$. Fig.(11) defines some simple rooted trees.

Note that each vertex $v_{i}$ of the rooted tree corresponds to an integration variable $x_{i}$, and that an edge connecting $v_{j}$ to $v_{i}$ towards the root indicates that the $x_{j}$ integration is nested in the $x_{i}$ integration. Integration variables which correspond to vertices which are not connected by an edge correspond to disjoint integrations.

For the rooted trees defined in Fig.(1) we find the following analytic expressions:

$$
\begin{aligned}
x_{t_{1}}(c) & =\int_{0}^{\infty} \frac{y^{-\epsilon}}{y+c} d y, \\
x_{t_{2}}(c) & =\int_{0}^{\infty} \frac{y^{-\epsilon} x_{t_{1}}(y)}{y+c} d y, \\
x_{t_{3_{1}}}(c) & =\int_{0}^{\infty} \frac{y^{-\epsilon} x_{t_{2}}(y)}{y+c} d y, \\
x_{t_{3_{2}}}(c) & =\int_{0}^{\infty} \frac{y^{-\epsilon} x_{t_{1}}(y) x_{t_{1}}(y)}{y+c} d y .
\end{aligned}
$$




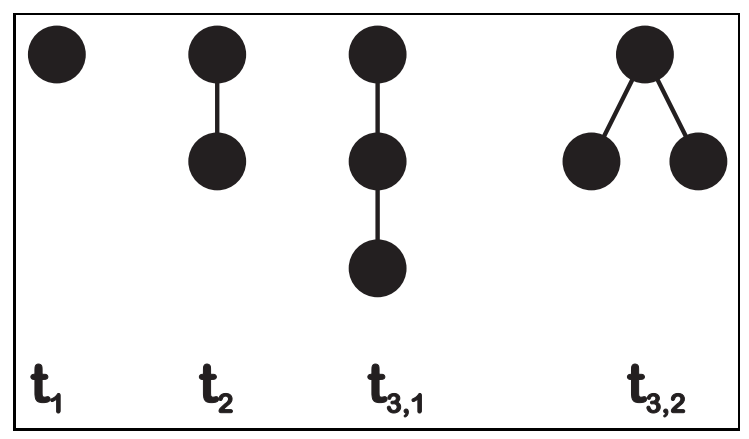

Figure 1: A toy model realizing rooted trees. We define the first couple of rooted trees $t_{1}, t_{2}, t_{3_{1}}, t_{3_{2}}$. The root is always drawn as the uppermost vertex. $t_{2}$ gives rise to the function $x_{2}(c)$.

Note that $x_{2}(c) \equiv x_{t_{2}}(c)$.

\section{The Hopf algebra $\mathcal{H}_{R}$}

The previous remarks motivate to introduce a Hopf algebra based on rooted trees. We still postpone all formal definitions to the next section and simply note that a rooted tree $t$ is a connected and simply-connected set of oriented edges and vertices such that there is precisely one distinguished vertex with no incoming edge. This vertex is called the root of $t$. Further, every edge connects two vertices and the fertility $f(v)$ of a vertex $v$ is the number of edges outgoing from $v$.

We consider the algebra of polynomials over $\mathbf{Q}$ in rooted trees.

Note that for any rooted tree $t$ with root $r$ we have $f(r)$ trees $t_{1}, \ldots, t_{f(r)}$ which are the trees attached to $r$.

Let $B_{-}$be the operator which removes the root $r$ from a tree $t$ :

$$
B_{-}: t \rightarrow B_{-}(t)=t_{1} t_{2} \ldots t_{f(r)} .
$$

Fig.(2) gives an example.

Let $B_{+}$the operation which maps a monomial of $n$ rooted trees to a new rooted tree $t$ which has a root $r$ with fertility $f(r)=n$ which connects to the $n$ roots of $t_{1}, \ldots, t_{n}$.

$$
B_{+}: t_{1} \ldots t_{n} \rightarrow B_{+}\left(t_{1} \ldots t_{n}\right)=t .
$$

This is clearly the inverse to the action of $B_{-}$.

One has

$$
\left.\left.B_{+}\left(B_{-}(t)\right)\right)=B_{-}\left(B_{+}(t)\right)\right)=t
$$

for any rooted tree $t$. Fig.(阿) gives an example. We further set $B_{-}\left(t_{1}\right)=1$, $B_{+}(1)=t_{1}$. 


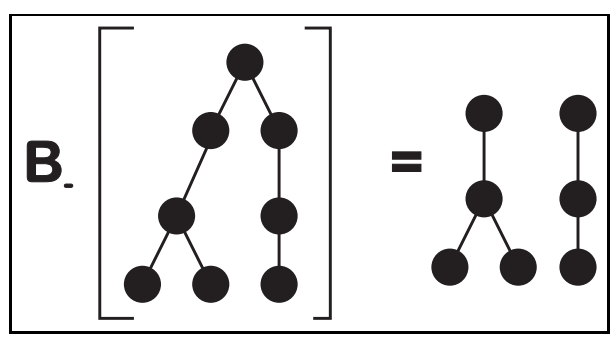

Figure 2: The action of $B_{-}$on a rooted tree.

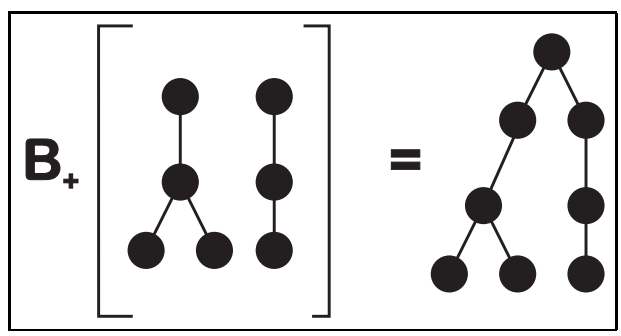

Figure 3: The action of $B_{+}$on a monomial of trees.

We will introduce a Hopf algebra on such rooted trees by using the possibility to cut such trees in pieces. We start with the most elementary possibility. An elementary cut is a cut of a rooted tree at a single chosen edge, as indicated in Fig.(4). We will formalize all these notions in the next section. By such a cutting procedure, we will obtain the possibility to define a coproduct in a moment, as we can use the resulting pieces on either side of the coproduct.

But before doing so we finally introduce the notion of an admissible cut, also called a simple cut. It is any assignment of elementary cuts to a rooted tree

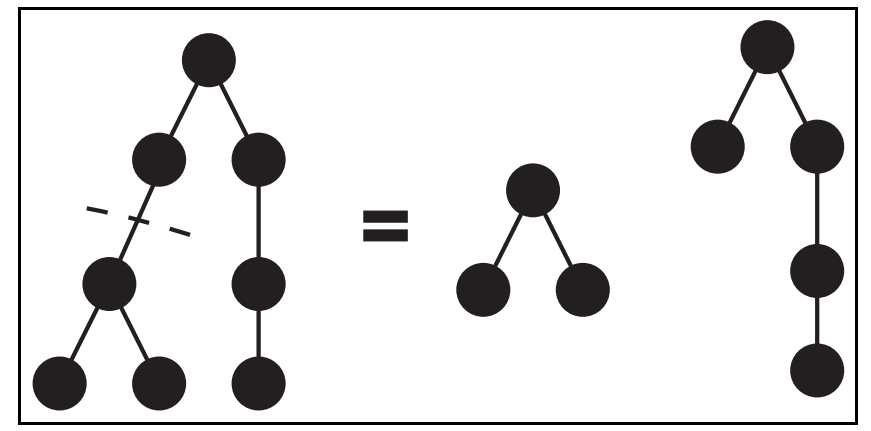

Figure 4: An elementary cut splits a rooted tree $t$ into two components $t_{1}, t_{2}$. 


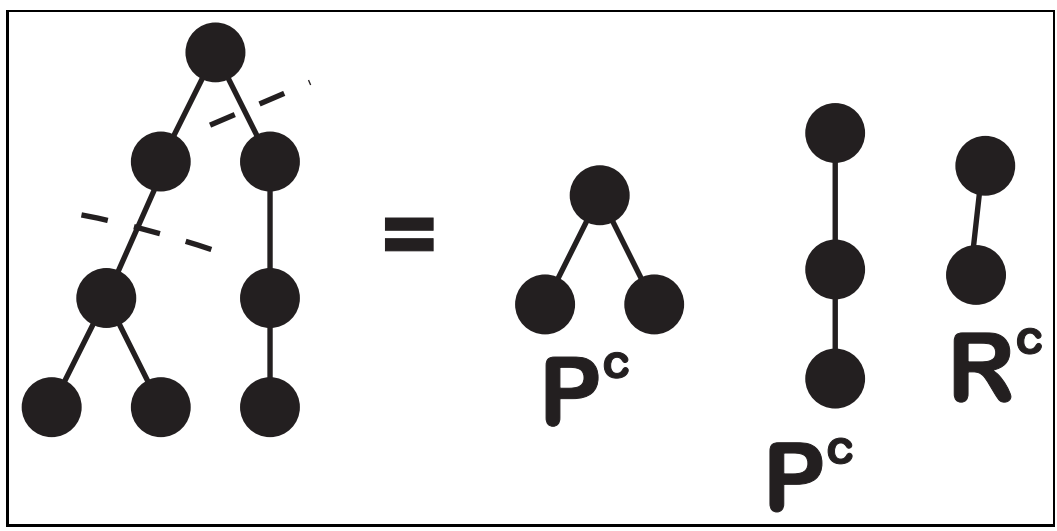

Figure 5: An admissible cut $C$ acting on a tree $t$. It produces a monomial of trees. One of the factors, $R^{C}(t)$, contains the root of $t$.

$t$ such that any path from any vertex of the tree to the root has at most one elementary cut. Fig.(5) gives an example.

An admissible cut $C$ maps a tree to a monomial in trees. If the cut $C$ contains $n$ elementary cuts, it induces a map

$$
C: t \rightarrow C(t)=\prod_{i=1}^{n+1} t_{j_{i}} .
$$

Note that precisely one of these trees $t_{j_{i}}$ will contain the root of $t$. Let us denote this distinguished tree by $R^{C}(t)$. The monomial which is delivered by the $n-1$ other factors is denoted by $P^{C}(t)$.

The definitions of $C, P, R$ can be extended to monomials of trees in the obvious manner, by choosing a cut $C^{i}$ for every tree $t_{j_{i}}$ in the monomial:

$$
\begin{aligned}
C\left(t_{j_{1}} \ldots t_{j_{n}}\right) & :=C^{1}\left(t_{j_{1}}\right) \ldots C^{n}\left(t_{j_{n}}\right), \\
P^{C}\left(t_{j_{1}} \ldots t_{j_{n}}\right) & :=P^{C^{1}}\left(t_{j_{1}}\right) \ldots P^{C^{n}}\left(t_{j_{n}}\right), \\
R^{C}\left(t_{j_{1}} \ldots t_{j_{n}}\right) & :=R^{C^{1}}\left(t_{j_{1}}\right) \ldots R^{C^{n}}\left(t_{j_{n}}\right) .
\end{aligned}
$$

We have now collected a sufficient amount of structure to define a Hopf algebra on rooted trees. Our aim is to see the correspondence between the Hopf algebra formulated on rooted trees and the generation of a local counterterm for the functions $x_{t}(c)$ introduced above, and finally to see the correspondence between the Hopf algebra of rooted trees and the Hopf algebra of the previous section.

Before we define the Hopf algebra of rooted trees, we leave it as an exercise to the reader to convince himself that any admissible cut in a rooted tree determines in the representation on functions $x_{t}(c)$ a divergent subintegration, and 
that vice versa any divergent subintegration corresponds to an admissible cut. For example, the single cut possible at $x_{2}(c) \equiv x_{t_{2}}(c)$ corresponds to the single divergent subintegration in this function.

Let us now establish the Hopf algebra structure. Following [1] we define the counit and the coproduct. The counit $\epsilon: \mathcal{A} \rightarrow \mathbf{Q}$ is simple:

$$
\epsilon(X)=0
$$

for any $X \neq e$,

$$
\epsilon(e)=1 \text {. }
$$

The coproduct $\Delta$ is defined by the equations

$$
\begin{aligned}
\Delta(e) & =e \otimes e \\
\Delta\left(t_{1} \ldots t_{n}\right) & =\Delta\left(t_{1}\right) \ldots \Delta\left(t_{n}\right) \\
\Delta(t) & =t \otimes e+\left(i d \otimes B_{+}\right)\left[\Delta\left(B_{-}(t)\right)\right],
\end{aligned}
$$

which defines the coproduct on trees with $n$ vertices iteratively through the coproduct on trees with a lesser number of vertices.

The reader should work out the examples in Fig.(6) himself. One checks coassociativity of $\Delta[1]$. Also, we will give a formal proof in the next section.

The following statement follows directly from the results in the next section, but it is instructive to prove it here by elementary means to make contact with the previous section. We claim that the coproduct can be written as

$$
\Delta(t)=e \otimes t+t \otimes e+\sum_{\text {adm. cuts } C \text { of } t} P^{C}(t) \otimes R^{C}(t) .
$$

Proof. The result is true for the tree $t_{1}$ having only one vertex. The induction is on the number of vertices. We use that $B_{-}(t)$ has $n$ vertices if $t$ has $n+1$. Thus,

$$
\begin{aligned}
\Delta(t)= & t \otimes e+\left(i d \otimes B_{+}\right) \Delta\left(B_{-}(t)\right) \\
= & t \otimes i d+\left(i d \otimes B_{+}\right)\left(e \otimes B_{-}(t)+B_{-}(t) \otimes e\right. \\
& \left.+\sum_{\text {adm. cuts } C \text { of } B_{-}(t)} P^{C}\left(B_{-}(t)\right) \otimes R^{C}\left(B_{-}(t)\right)\right) \\
= & t \otimes e+e \otimes B_{+}\left(B_{-}(t)\right)+B_{-}(t) \otimes \delta_{1} \\
& \left.+\sum_{\text {adm. cuts } C \text { of } B_{-}(t)} P^{C}\left(B_{-}(t)\right) \otimes B_{+}\left(R^{C}\left(B_{-}(t)\right)\right)\right) \\
= & t \otimes e+e \otimes t \\
& \left.+\sum_{\text {adm. cuts } C \text { of } t} P^{C}(t) \otimes R^{C}(t)\right) .
\end{aligned}
$$




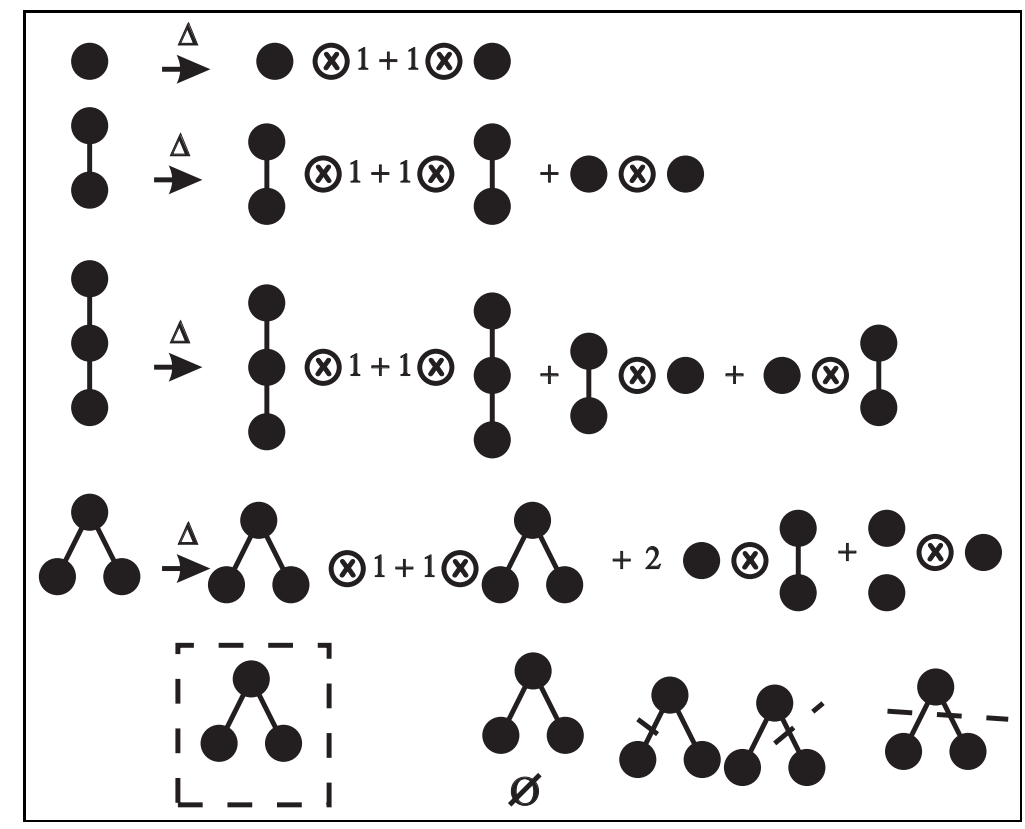

Figure 6: The coproduct. We work it out for the trees $t_{1}, t_{2}, t_{3_{1}}, t_{3_{2}}$. For the latter, the last line gives explicitly the simple admissible cuts which were used in the construction of the coproduct. The first two terms are generated by the full admissible and the empty cut, while the last three terms are generated by proper admissible cuts. 
We used the fact that $B_{+} B_{-}=i d$ and that the only cut which distinguishes

$$
\sum_{\text {adm. cuts } C \text { of } B_{-}(t)} P^{C}\left(B_{-}(t)\right) \otimes B_{+}\left(R^{C}\left(B_{-}(t)\right)\right)
$$

from

$$
\sum_{\text {adm. cuts } C \text { of } t} P^{C}(t) \otimes R^{C}(t)
$$

is the cut which generates $B_{-}(t) \otimes \delta_{1}$.

Note that the above formula can be streamlined.

$$
\Delta(t)=\sum_{\text {adm. cuts } C \text { of } t}^{\prime} P^{C}(t) \otimes R^{C}(t)
$$

where the primed sum indicates that we include the empty cut and the full admissible cut in the definition of admissible cuts in the manner indicated in Fig.(6).

Any cut corresponds to a choice of a subset of edges on the set $t^{(1)}$ of all edges of a given rooted tree $t$. The empty cut $C=\emptyset$ corresponds to the empty set in this sense. Thus,

$$
\begin{aligned}
& P^{\emptyset}(t)=e, \\
& R^{\emptyset}(t)=t .
\end{aligned}
$$

The full admissible cut $C_{f}$ is defined by the complementary result:

$$
\begin{aligned}
& P^{C_{f}}(t)=t, \\
& R^{C_{f}}(t)=e .
\end{aligned}
$$

It can be regarded as a cut $c$ on the one new edge of $B_{+}(t)$, defined as the intersection $c=\left\{t^{(1)} \cap B_{+}(t)^{(1)}\right\}$. Note that

$$
P^{C_{f}}(t) \otimes R^{C_{f}}(t)=\left(i d \otimes B_{-}\right)\left[P^{c}\left(B_{+}(t)\right) \otimes R^{c}\left(B_{+}(t)\right)\right]
$$

with the cut $c$ determined as above. In Fig.(6) we indicate the full cut $C_{f}\left(t_{3_{2}}\right)$ by a dashed box around the rooted tree $P^{C_{f}}\left(t_{3_{2}}\right)$.

The coproduct introduced here is linear in rooted trees in the right factor and polynomial in the left as one clearly sees in Eq.(51). This is a fundamental property shared with the coproduct of the previous section. We will explore this fact in some detail soon.

Up to now we have established a bialgebra structure. It is actually a Hopf algebra. Following [1] we find the antipode $S$ as

$$
\begin{aligned}
S(e) & =e \\
S(t) & =-t-\sum_{\text {adm. cuts } C \text { of } t} S\left[P^{C}(t)\right] R^{C}(t),
\end{aligned}
$$


and one immediately checks that

$$
\begin{aligned}
m[(S \otimes i d) \Delta(t)] & =t+S(t)+\sum_{\text {adm. cuts } C \text { of } t} S\left[P^{C}(t)\right] R^{C}(t) \\
& =0=\epsilon(t) .
\end{aligned}
$$

To show that $m[(i d \otimes S) \Delta(t)]=0$ one uses induction on the number of vertices [1].

We mentioned already that a cut on a tree $t$ is given by a subset of the set $t^{(1)}$ of the set of edges of $t$. So far, we allowed for a restricted class of subsets, corresponding to admissible cuts. We actually enlarged the set already and considered the set $B_{+}(t)^{(1)}$ of all edges of $B_{+}(t)$, to construct the full admissible cut. We now consider all cuts corresponding to this set, that is all possible subsets of $B_{+}(t)^{(1)}$, including the empty set. These subsets fall in two classes, one which contains the edge $c(t)=t^{(1)} \cap B_{+}(t)^{(1)}$, one which does not contain this edge.

Cuts corresponding to the first class we call full cuts, cuts not containing this distinguished edge we call normal cuts. Thus, the empty cut is a normal cut. Non-empty normal cuts are also called proper cuts. Note that for a given normal cut $C \subset t^{(1)}$ and the corresponding full cut $\{C, c(t)\}=C \cap c(t)$ we have

$$
\begin{aligned}
& P^{C}(t)=P^{\{C, c(t)\}}(t), \\
& R^{C}(t)=R^{\{C, c(t)\}}(t),
\end{aligned}
$$

while $n_{\{C, c(t)\}}=n_{C}+1$, where $n_{C}$ is the cardinality of the set $C$.

Let us give yet another formula to write the antipode, which one easily derives using induction on the number of vertices:

$$
S(t)=\sum_{\text {all full cuts } C \text { of } t}(-1)^{n_{C}} P^{C}(t) R^{C}(t) .
$$

This time, we have a non-recursive expression, summing over all full cuts $C$, relaxing the restriction to admissible cuts. We introduced full cuts so that the overall sign agrees with the number of cuts employed.

Note that we have for all $t \neq e$

$$
m[(S \otimes i d) \Delta(t)]=\sum_{\text {all cuts } C \text { of } t}(-1)^{n_{C}} P^{C}(t) R^{C}(t)=0=\epsilon(t),
$$

as each cut appears twice, either as a full cut or as a normal cut, with opposite sign, due to the fact that the cardinality of $\{C, c(t)\}$ extends the cardinality of $C$ by one.

By now we have established a Hopf algebra on rooted trees. It is instructive to calculate the coproduct and antipode for some simple trees. Figs.(6), (7) give examples. Note that in Fig.(7) we represented cuts as boxes. This is possible 


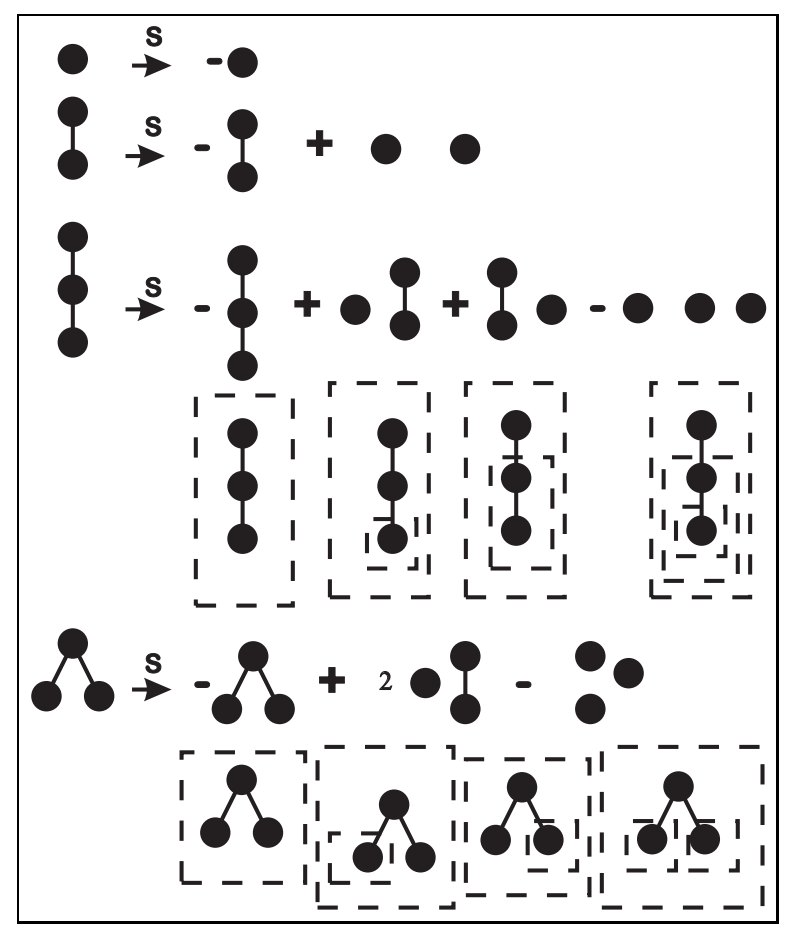

Figure 7: The antipode for some simple trees. Introducing full cuts, we find a very convenient way to express it using boxes for cuts. The sign for each term can be easily memorized as $(-1)^{n_{c}}$, where $n_{c}$ is the number of full cuts.

in a unique way, as each cut on a simply connected rooted tree can be closed in the plane to a box without further intersecting the tree and so that the root is in the exterior of the box.

At this time we can make contact to the previous section. We note that the sum of the two trees with three vertices behaves under the coproduct as the element $\delta_{3}$ in the first section. Defining $\delta_{1}:=t_{1}, \delta_{2}:=t_{2}, \delta_{3}:=t_{3_{1}}+t_{3_{2}}$, we find

$$
\begin{aligned}
& \Delta\left(\delta_{1}\right)=\delta_{1} \otimes e+e \otimes \delta_{1} \\
& \Delta\left(\delta_{2}\right)=\delta_{2} \otimes e+e \otimes \delta_{2}+\delta_{1} \otimes \delta_{1} \\
& \Delta\left(\delta_{3}\right)=\delta_{3} \otimes e+e \otimes \delta_{3}+3 \delta_{1} \otimes \delta_{2}+\delta_{2} \otimes \delta_{1}+\delta_{1}^{2} \otimes \delta_{1},
\end{aligned}
$$

in accordance with Eq.(10) in the previous section. This is no accident, as we will soon see.

The reader should also check the formulas for the coproduct and antipode on examples himself.

It is now not difficult to employ this Hopf algebra to regain the local counterterms for the toy model. By construction, subdivergent sectors correspond to 


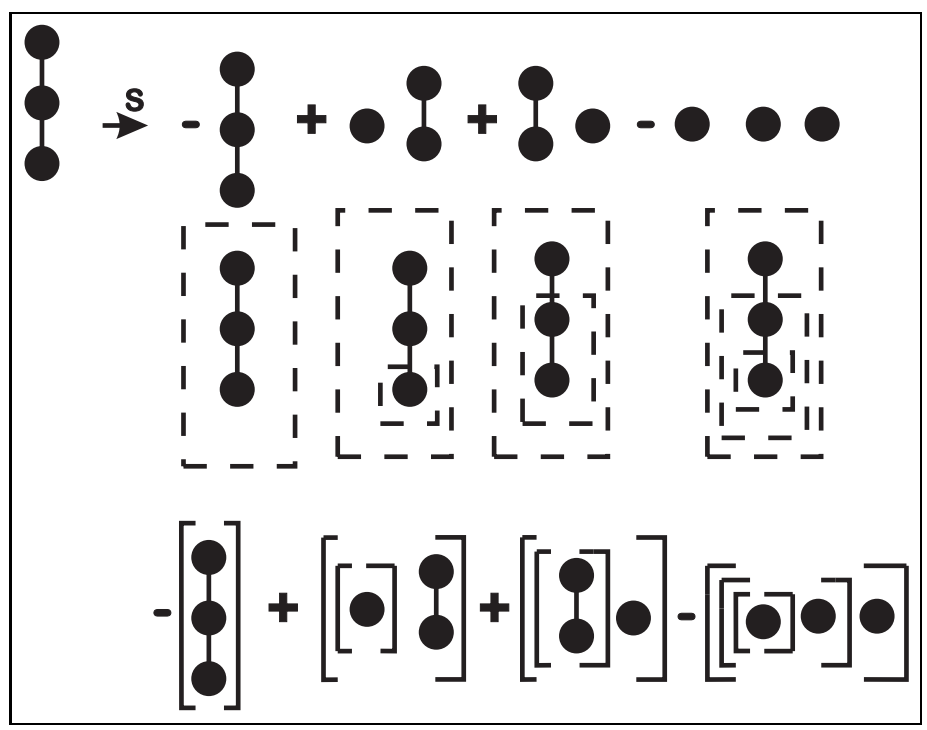

Figure 8: Cuts induce a bracket structure on trees. Exploring this fact, the toy model can be easily renormalized using the Hopf algebra structure of rooted trees.

admissible cuts. Also, forests in the sense of renormalization theory are in one to one correspondence with arbitrary cuts, with full cuts corresponding to full forests, and normal cuts to normal forests. This allows to recover local counterterms from the formula for the antipode in our Hopf algebra. The recursive and the non-recursive manner to write the antipode give rise to two equivalent formulas for the local counterterm, as it is standard in renormalization theory [4].

To see all this, note that any cut defines a natural bracket structure on $P^{c}(t)$. In a moment we will see how this fact allows to introduce various different renormalization schemes $R$.

It is instructive to come back to the toy model. Fig.(8) summarizes how the standard notions of renormalization theory derive from the Hopf algebra of rooted trees.

In Fig.(8) we see the tree $t_{3_{1}}$, corresponding to the function $x_{t_{3_{1}}}(c)$. The antipode

$$
S\left(t_{3_{1}}\right)=-t_{3_{1}}+t_{1} t_{2}+t_{2} t_{1}-t_{1} t_{1} t_{1}
$$

derives from full cuts which induce the following bracket structure on toy model functions:

$$
-\left[x_{t_{3_{1}}}(c)\right]+\left[\left[x_{t_{2}}(c)\right] x_{t_{1}}(c)\right]+\left[\left[x_{t_{1}}(c)\right] x_{t_{2}}(c)\right]-\left[\left[\left[x_{t_{1}}(c)\right] x_{t_{1}}(c)\right] x_{t_{1}}(c)\right] .
$$

There is a certain freedom how to evaluate this bracket structure. Such a free- 
dom is always there in renormalization. It corresponds to a choice of renormalization map $R$ in the notation of [1], while in Collins textbook [4] it corresponds to the choice of the map $T$ which extracts the divergent part of a given expression.

As long as the evaluation of the bracket leaves the divergent part unchanged, it corresponds to a valid renormalization scheme, and we obtaine the finite renormalized Green function from the consideration of $m\left[(S \otimes i d) \Delta\left(t_{3_{1}}\right)\right]$, which gives rise to the following expression by summing over brackets induced by full and normal cuts

$$
\begin{aligned}
x_{t_{3_{1}}}^{R}(c) & =x_{t_{3_{1}}}(c)-\left[x_{t_{2}}(c)\right] x_{t_{1}}(c)-\left[x_{t_{1}}(c)\right] x_{t_{2}}(c)+\left[\left[x_{t_{1}}(c)\right] x_{t_{1}}(c)\right] x_{t_{1}}(c) \\
& -\left[x_{t_{3_{1}}}(c)\right]+\left[\left[x_{t_{2}}(c)\right] x_{t_{1}}(c)\right]+\left[\left[x_{t_{1}}(c)\right] x_{t_{2}}(c)\right]-\left[\left[\left[x_{t_{1}}(c)\right] x_{t_{1}}(c)\right] x_{t_{1}}(c)\right] .
\end{aligned}
$$

It is not to difficult to check the finiteness of this expression for the typical choices of renormalization schemes, on shell,

$$
\left[x_{t}(c)\right]=x_{t}(1),
$$

minimal subtraction,

$$
\left[x_{t}(c)\right]=\operatorname{PolePart}_{\epsilon}\left(x_{t}(1)\right),
$$

or BPHZ type schemes.

Note that the only reason that the renormalized function $x_{t}^{R}(c)$ does not vanish identically is that full cuts involve one more bracket evaluation than normal cuts. As the bracket evaluation respects the divergent part, it is clear that functions $x_{t}^{R}(c)$ must be finite, due to the very fact that $m[(S \otimes i d) \Delta(t)]$ involves a sum over all cuts in pairs of normal cuts and associated full cuts. This gives rise to a sum of pairs of contributions, each pair being a difference $X-[X]$ between an analytic contribution $X$ and its bracket evaluation. Thus, as long as the bracket evaluation respects the divergent part of $X$, we will obtain a finite result for $x_{t}^{R}(c)$.

Let us now turn away from toy models and address QFTs. By its very definition, pQFT deals with the calculation of Feynman diagrams. As such, it confronts the problem of the presence of ultraviolet divergences in the diagrams. Hence, the diagrams refer to ill-defined analytic quantities. This is reflected by the fact that the analytic expressions provided by the diagrams become Laurent series in a regularization parameter. The presence of pole terms in this Laurent series then indicates the presence of UV-divergences in the first place.

Equivalently, in the BPHZ spirit, we can Taylor expand the integrand in external momenta and would find that the first few terms are ill-defined quantities.

The art of obtaining meaningful physical quantities from these Laurent series is known as renormalization. It is in this process that we will find the Hopf algebra structure realized. 


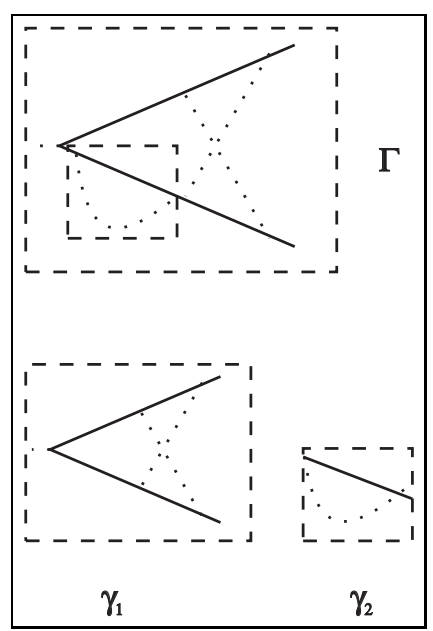

Figure 9: This Feynman graph $\Gamma$ behaves in the same manner as the toy function $x_{2}(c)$. It contains a subdivergent graph $\gamma_{2} \subset \Gamma$, contained in the smaller box. This subdivergence sits in a graph $\gamma_{1}$ which we obtain if we shrink $\gamma_{2}$ to a point in $\Gamma, \gamma_{1}=\Gamma / \gamma_{2}$. To get a local counterterm, we follow the same steps as before: we replace the divergent subgraph $\gamma_{2}$ by its subtracted renormalized form $\gamma_{2}^{R}=\gamma_{2}-\left[\gamma_{2}\right]$, and can calculate the local counterterm for $\Gamma$ from the resulting expression.

Let us recall a few basic properties of renormalization. Feynman diagrams consist of edges (propagators) and vertices of different types. To each such type we can assign a weight, and to a Feynman diagram we can assign an integral weight called degree of divergence which can be calculated from the dimension of spacetime, the numbers of closed cycles in the Feynman diagram, and the weights of its propagators and vertices.

One finds that the analytic expression provided by a Feynman diagram under consideration provides UV-divergences if and only if its degree of divergence $\omega$ is $\geq 0$. One speaks of logarithmic, linear, quadratic, ... divergences for $\omega=0,1,2, \ldots$.

A Feynman diagram usually contains subdiagrams, which have their own degree of divergence, and thus might provide UV-divergent analytic expressions by themselves. All these divergences are to be compensated by local counterterms, which are to be calculated from a Feynman graph and its divergent subgraphs.

Fig.(9) shows how the previous discussion extends to a Feynman graph with one subdivergent graph.

It is the main result of [1] that the transition from a bare Feynman diagram to its local counterterm, and to the renormalized Feynman graph, is described by a Hopf algebra structure. In this paper, we have changed the notation of 


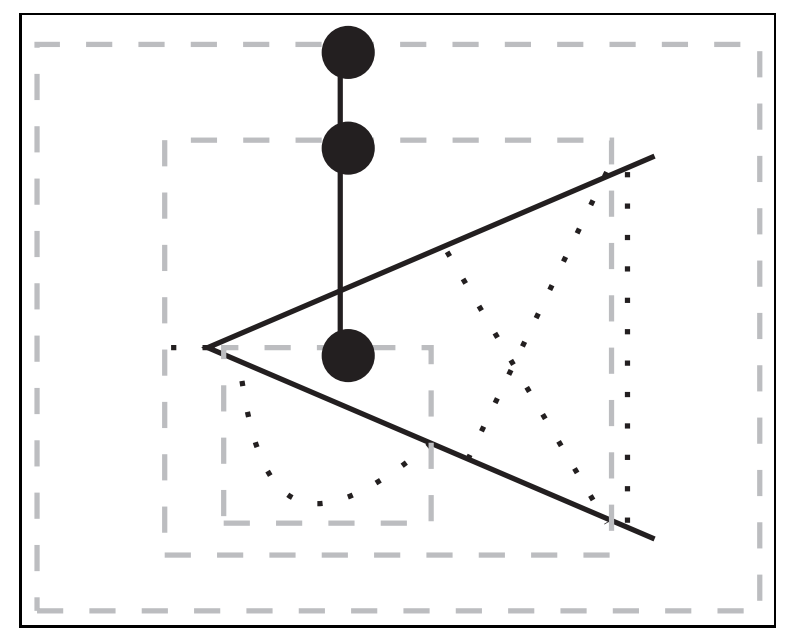

Figure 10: Rooted trees from Feynman diagrams. They are in one-to-one correspondence with the parenthesized words of [1]. In this example it is the rooted tree $t_{3_{1}}$ which is associated to the configuration of subdivergences given by the graph.

[1] and formulate the Hopf algebra on rooted trees. A glance at Fig. (10) shows how to assign a rooted tree to a Feynman graph with subdivergences. This is possible in a unique manner as long as all subdivergences of the Feynman graph are either disjoint or nested [1]. In such circumstances, we can associate a unique rooted tree to the graph. If the subdivergences are overlapping, the renormalization will nevertheless follow the combinatorics dictated by the Hopf algebra of rooted trees. In such circumstances, a Feynman graph corresponds to a sum of rooted trees [5, 1]. We will comment on this fact in an appendix.

Each Feynman diagram $\Gamma$ furnishes a tree whose vertices are decorated by Feynman graphs $\gamma \subset \Gamma$ which are free of subdivergences themselves. These decorations correspond to the letters in the parenthesized words of [1].

Note that the set of all admissible cuts is by construction in one to one correspondence with the set of all superficially divergent subgraphs of $\Gamma$. Further the set of all cuts is in one to one correspondence with the set of all forests in the sense of renormalization theory.

This is the main result of [1]. The antipode of the Hopf algebra $\mathcal{A}_{Q F T}$ delivers the $Z$-factor of a Feynman diagram.

At this stage, we can summarize the results in 1 il using the language of the Hopf algebra of rooted trees. This is done in Fig.(11). Note that we even do not have to specify the renormalization scheme, but that the cuts used in the definition of the antipode on rooted trees extend to forests, so that we can apply any chosen renormalization prescription to evaluate the content of these forests. 
Thus, as before in the toy model, each cut corresponds to the operation $T$ in Collins book [4], some operation which extracts the divergence of the expression on which it acts. As we mentioned already, this operation was called $R$ in [1].

At this point, we succeeded in deriving the renormalization procedure from the Hopf algebra of rooted trees. The attentive reader will have noticed that we ignored overlapping divergences in our discussion. As promised, we will discuss them in an appendix, where it is shown how to assign a unique sum of rooted trees to any graph containing overlapping divergences, to which then our previous considerations apply.

For us, these considerations of renormalization and the underlying Hopf algebra of rooted trees are sufficient motivation to get interested in this Hopf algebra. We will continue our exploration of this subject by showing how it relates to the Hopf algebra of the first section. We saw some of these relations already, and now continue to make this more precise. 


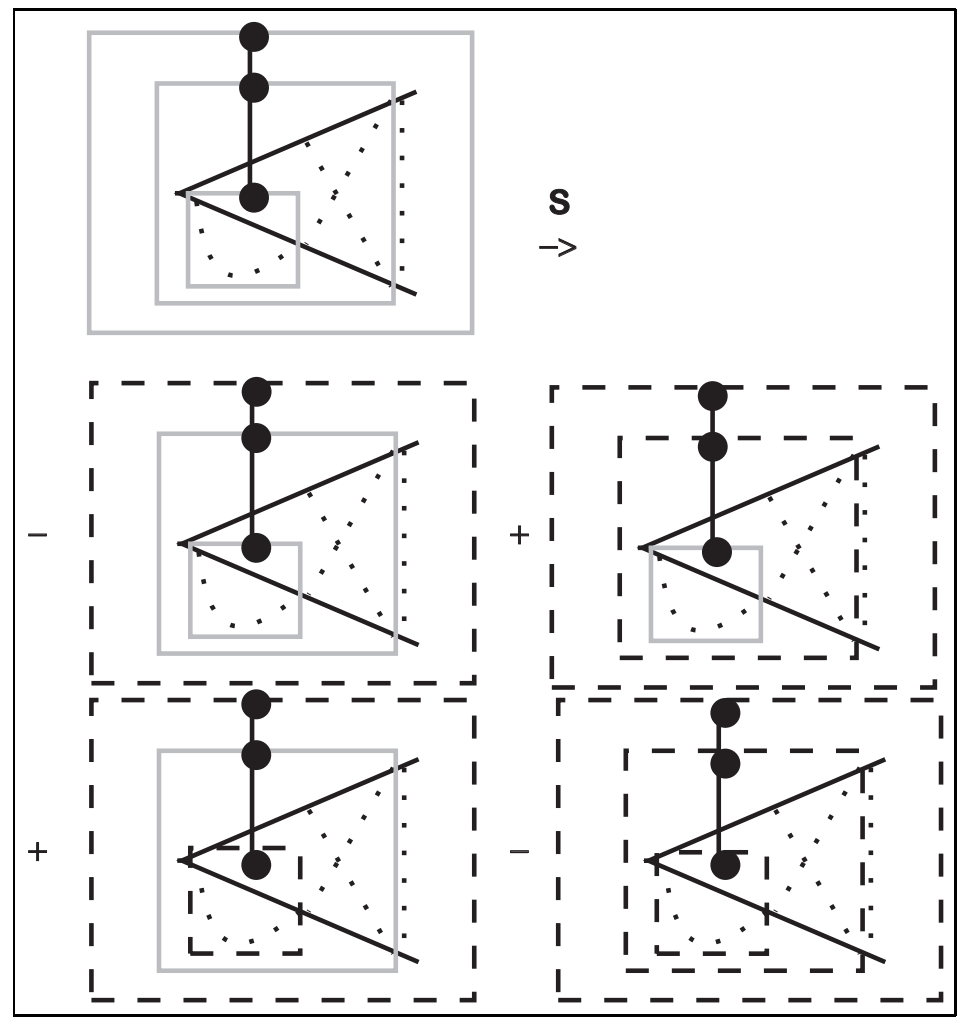

Figure 11: The steps involved in the process of renormalization are governed by the Hopf algebra on rooted trees, as this figure clearly exhibits. We indicate how the Hopf algebra of rooted trees acts on the Feynman diagrams. Subgraphs are indicated by grey rectangles and determine the tree structure, forests corresponding to cuts generated by the antipode of the associated rooted tree are given as dashed black rectangles. 


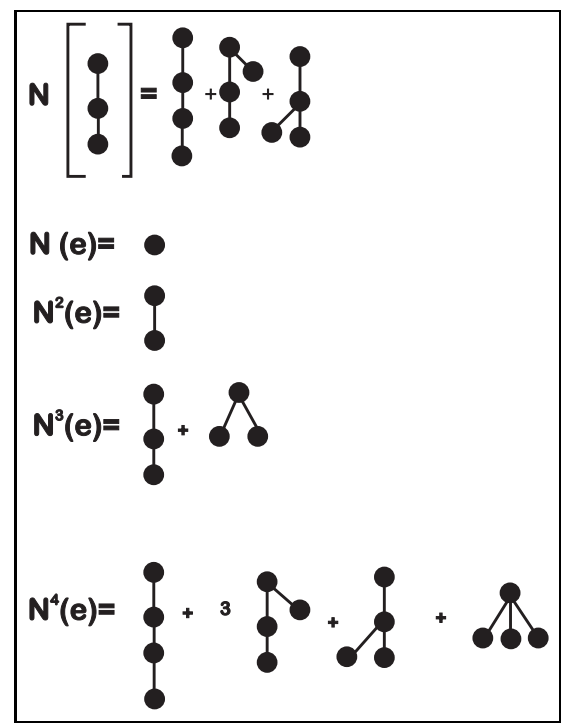

Figure 12: The operator $N$ and the elements $\delta_{k}$.

\section{The relation between $\mathcal{H}_{R}$ and $\mathcal{H}_{T}$}

Recall the relations

$$
\begin{aligned}
& \Delta\left(\delta_{1}\right)=\delta_{1} \otimes e+e \otimes \delta_{1} \\
& \Delta\left(\delta_{2}\right)=\delta_{2} \otimes e+e \otimes \delta_{2}+\delta_{1} \otimes \delta_{1} \\
& \Delta\left(\delta_{3}\right)=\delta_{3} \otimes e+e \otimes \delta_{3}+3 \delta_{1} \otimes \delta_{2}+\delta_{2} \otimes \delta_{1}+\delta_{1}^{2} \otimes \delta_{1},
\end{aligned}
$$

which indicate an intimate connection to the Hopf algebra $\mathcal{H}_{T}$ introduced in the first section. To find the general relation between the two Hopf algebras under consideration we first introduce naturally grown forests $\delta_{k}$.

To this end, we consider an operator $N$ which maps a tree $t$ with $n$ vertices to a sum $N(t)$ of $n$ trees $t_{i}$, each having $n+1$ vertices, by attaching one more outgoing edge and vertex to each vertex of $t$, as in Fig.(12). The root remains the same in this operation.

Now we define

$$
\delta_{k}:=N^{k}(e)
$$

so that $\delta_{k+1}=N\left(\delta_{k}\right)$. On products of trees $N$ will act as a derivation, comparable to the derivation $D$ introduced in Eq.(29).

In Fig. (12) we see the first few elements $\delta_{k}$. Note that there are non-trivial multiplicities as in $\delta_{4}$.

Let

$$
\left[X, \delta_{n}\right]=\delta_{n+1},
$$




$$
\left[Y, \delta_{n}\right]=n \delta_{n} .
$$

The following result, which is a trivial consequence of the results in the next section, initiated this paper:

i) With the coproduct of $\mathcal{H}_{R}$, the $\delta_{k}$ span a closed Hopf subalgebra of $\mathcal{H}_{R}$.

ii)

$$
\begin{aligned}
\Delta\left(\delta_{n}\right) & =e \otimes \delta_{n}+\delta_{n} \otimes e+R_{n-1} \\
R_{0} & =0 \\
R_{1} & =\delta_{1} \otimes \delta_{1} \\
R_{k} & =\left[X \otimes e+e \otimes X, R_{k-1}\right]+k \delta_{1} \otimes \delta_{k}+\left[\delta_{1} \otimes Y, R_{k-1}\right] .
\end{aligned}
$$

The proof follows from the results in the next section, but it is instructive to investigate directly the compatibility of the operation of natural growth and the notion of an admissible cut: We note that $\delta_{n}$ is a sum of trees: $\delta_{n}=\sum_{i} t_{i}$, say. Thus,

$$
\Delta\left(\delta_{n}\right)=e \otimes \delta_{n}+\delta_{n} \otimes e+\sum_{i} \sum_{\text {all cuts } C^{i} \text { of } t_{i}} P^{C^{i}}\left(t_{i}\right) \otimes R^{C^{i}}\left(t_{i}\right)
$$

Hence we can write, with the same $t_{i}$ as before,

$$
\begin{aligned}
\Delta\left(\delta_{n+1}\right)= & e \otimes \delta_{n+1}+\delta_{n+1} \otimes e \\
& +\sum_{i} \sum_{\text {all cuts } C^{i} \text { of } t_{i}}\left\{N\left[P^{C^{i}}\left(t_{i}\right)\right] \otimes R^{C^{i}}\left(t_{i}\right)\right. \\
& +\left(P^{C^{i}}\left(t_{i}\right) \otimes N\left[R^{C^{i}}\left(t_{i}\right)\right]\right\} \\
& +n \delta_{1} \otimes \delta_{n} \\
& +\sum_{i} \sum_{\text {all cuts } C^{i} \text { of } t_{i}} l\left[R^{C^{i}}\left(t_{i}\right)\right] \delta_{1} P^{C^{i}}\left(t_{i}\right) \otimes R^{C i}\left(t_{i}\right),
\end{aligned}
$$

where $\mathrm{l}(\mathrm{t})$ gives the number of vertices of a tree $t$. Thus, we decomposed the cuts at the components of $\delta_{n+1}$ in four classes: either the edge to the new grown vertex is not cut, then we will have natural growth on either the former $P^{C}$ or $R^{C}$ part. Thus, the first two contributions deliver the operator $N$ on either side of the tensorproduct. Or, for the remaining two cases, the edge to the new grown vertex is cut. These cases will always have a factor $\delta_{1}$ on the lhs of the tensorproduct.

In these cases, it either was grown from the former $R^{C}$ part (admissibility of cuts forbid that it was grown from the $P^{C}$ part), or it was grown from the whole uncut former $\delta_{n}$, which gives the term $n \delta_{1} \otimes \delta_{n}$.

Hence we have decomposed the cuts possible at the trees of $\delta_{n+1}$ in terms of the cuts at the trees of $\delta_{n}$. 


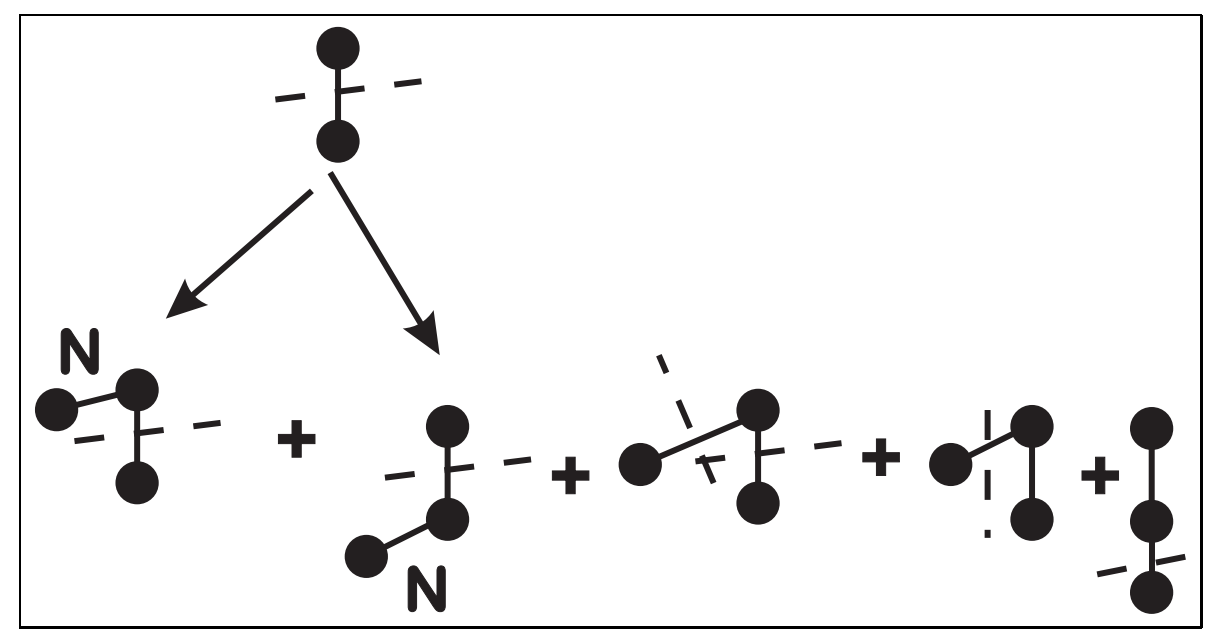

Figure 13: The decomposition of the cuts at $\delta_{n+1}$ in terms of the cuts at $\delta_{n}$ and the operator $N$. The first two terms of the bottom line indicate natural growth on the $P^{C}$ or the $R^{C}$ part. The third term gives the contribution for the case that the natural growth carries a cut itself. This can only happen at the $R^{C}$ part, due to admissibility of cuts. The last two terms are generated by the remaining possibility that the natural growth carries the sole cut.

Fig.(13) gives an instructive example. To finally prove the result, we note the following identities

$$
\begin{aligned}
N\left(\delta_{i_{1}} \ldots \delta_{i_{k}}\right) & =\left[X, \delta_{i_{1}} \ldots \delta_{i_{k}}\right] \\
l\left(\delta_{k}\right) \delta_{k} & =k \delta_{k}=\left[Y, \delta_{k}\right],
\end{aligned}
$$

where we note that $l\left[\delta_{k}\right]=k$ is well-defined, as $\delta_{k}$ is a homogenous combination of trees with $k$ vertices.

At this stage, we begin to see a fundamental connection between the process of renormalization and the results of [2]. Thus, we will now set out to define the Hopf algebra of rooted trees more formally and repeat the analysis of [2] for it.

We shall formalize the simplest example from the last section as the Hopf algebra of rooted trees, and extend many of the results of the first section to this more involved case.

By a rooted tree $T$ we mean a finite, connected, simply connected, one dimensional simplicial complex with a base point $* \in T^{(0)}=\{$ set of vertices of $T\}$. This base point is called the root. By the degreee of the tree we mean

$$
\operatorname{deg}(T)=\operatorname{Card} T^{(0)}=\# \text { of vertices of } T .
$$

For each $n$ we have a finite set of rooted trees $T$ with $\operatorname{deg}(T)=n$ where we only consider isomorphism classes of trees and choose a representative in each 
isomorphism class. Thus for $n=1$ we have one element $t_{1} \equiv *$, for $n=2$ we also have only one, $t_{2}$, and for $n=3$ we have two, $t_{3_{1}}$ and $t_{3_{2}}$, all defined in Fig.(11).

By a simple cut of a tree $T$ we mean a subset $c \subset T^{(1)}$ of the set of edges of $T$ such that,

for any $x \in T^{(0)}$ the path $(*, x)$ only contains at most one element of $c$.

Thus what is excluded is to have two cuts of the same path or branch. Given a cut $c$ the new simplicial complex $T_{c}$ with $T_{c}^{(0)}=T^{(0)}$ and

$$
T_{c}^{(1)}=T^{(1)} \backslash c,
$$

is no longer connected, unless $c=\emptyset$. We let $R_{c}(T)$ be the connected component of $*$ with the same base point and call it the trunk, while for each other connected component, called a cut branch, we endow it with the base point which is the edge of the cut. We obtain in this way a set, with multiplicity, of finite rooted trees.

For each $n$ we let $\Sigma_{n}$ be the set of trees of degree $\leq n$, up to isomorphism, and let $\mathcal{H}_{n}$ be the polynomial commutative algebra generated by the symbols,

$$
\delta_{T}, T \in \Sigma_{n} .
$$

One defines a coproduct on $\mathcal{H}_{n}$ by,

$$
\Delta \delta_{T}=\delta_{T} \otimes 1+1 \otimes \delta_{T}+\sum_{c}\left(\prod_{P_{c}(T)} \delta_{T_{i}}\right) \otimes \delta_{R_{c}(T)},
$$

where the last sum is over all non trivial simple cuts $(c \neq \emptyset)$ of $T$, while the product $\prod_{P_{c}(T)}$ is over the cut branches, in accordance with Eq.(51).

Equivalently, one can write (69) as,

$$
\left.\Delta \delta_{T}=\delta_{T} \otimes 1+\sum_{c}\left(\prod_{P_{c}(T)} \delta_{T_{i}}\right) \otimes \delta_{R_{c}(T)},\right)
$$

where the last sum is over all simple cuts.

This defines $\Delta$ on generators and it extends uniquely as an algebra homomorphism,

$$
\Delta: \mathcal{H}_{n} \rightarrow \mathcal{H}_{n} \otimes \mathcal{H}_{n}
$$

Lemma 1. The coproduct $\Delta$ is coassociative.

Proof. It is enough to check the equality

$$
(1 \otimes \Delta) \Delta \delta_{T}=(\Delta \otimes 1) \Delta \delta_{T} \quad \forall T \in \Sigma_{n},
$$


one can do it directly by introducing the notion of a double cut of $T$, but we shall use instead the following map from $\mathcal{H}_{R}=\cup \mathcal{H}_{n}$ to $\mathcal{H}_{R}$,

$$
L\left(\delta_{T_{1}} \ldots \delta_{T_{m}}\right)=\delta_{T}, \forall T_{j} \in \Sigma=\cup \Sigma_{n}
$$

where $T$ is the pointed tree obtained by connecting a new base point $*$ to the base points of the pointed trees $T_{j}$. The map $L$ is the unique linear map from $\mathcal{H}_{R}$ to $\mathcal{H}_{R}$ satisfying (73). It agrees with the map $B_{+}$introduced in the previous section. Let us show that,

$$
\Delta \circ L=L \otimes 1+(\operatorname{id} \otimes L) \circ \Delta .
$$

Let $a=\delta_{T_{1}} \ldots \delta_{T_{m}}$ and $T$ be as in (73) so that $L(a)=\delta_{T}$. From (70), one gets,

$$
\Delta(L(a))-L(a) \otimes 1=\sum_{c} \prod_{P_{c}} \delta_{T_{i}^{\prime}} \otimes \delta_{R_{c}},
$$

where all simple cuts of $T$, (including $c=\emptyset$ ) are allowed. Moreover,

$$
\Delta(a)=\prod_{i=1}^{n}\left(\delta_{T_{i}} \otimes 1+\sum_{c_{i}} \prod_{P_{c_{i}}} \delta_{T_{i_{j}}^{\prime \prime}} \otimes \delta_{R_{c_{i}}}\right),
$$

where again all simple cuts $c_{i}$ of $T_{i}$ are allowed.

Let $t_{n}$ be the tree with base point $*$ and $n$ other vertices $v_{i}$ labelled from $i=1$ to $i=n$, all directly connected to the base point $*$. We view $t_{n}$ in an obvious way as a subgraph of the tree $T$, where the base points are the same and the vertex $v_{i}$ is the base point of $T_{i}$. Given a simple cut $c$ of $T$ one gets by restriction to the subgraph $t_{n} \subset T$ a cut of $t_{n}$, it is characterized by the subset $I \subset\{1, \ldots, n\}, I=\left\{i ;\left(*, v_{i}\right) \in c\right\}$. The simple cut $c$ is uniquely determined by the subset $I$ and for each $i \in I^{c}$, i.e. each branch $\left(*, v_{i}\right)$ of $t_{n}$ which is not cut, by the simple cut $c_{i}$ of $T_{i}$ given by the restriction of $c$ to this subgraph. Thus the simple cuts $c$ of $T$ are in one to one correspondence with the various terms of the expression (76), namely the $\prod_{k \in I} \delta_{T_{k}} \otimes 1 \prod_{i \in I^{c}} \prod_{P_{c_{i}}} \delta_{T_{i_{j}}^{\prime \prime}} \otimes \delta_{R_{c_{i}}}$.

The two sums match termwise and, applying id $\otimes L$ to (76) one gets,

$$
\Delta(L(a))=L(a) \otimes 1+(\mathrm{id} \otimes L) \Delta(a) .
$$

This is Eq. (50) of the previous section. (Note that $L(1)=\delta_{*}$ by definition.)

One has,

$$
\Delta \delta_{*}=\delta_{*} \otimes 1+1 \otimes \delta_{*}
$$

so that $\mathcal{H}_{1}$ is coassociative. Let us assume that $\mathcal{H}_{n}$ is coassociative and prove it for $\mathcal{H}_{n+1}$. It is enough to check $(72)$ for the generators $\delta_{T}$, with $\operatorname{deg}(T) \leq n+1$ one has $\delta_{T}=L\left(\delta_{T_{1}} \ldots \delta_{T_{m}}\right)=L(a)$ where the degree of all $T_{j}$ is $\leq n$, i.e. $a \in \mathcal{H}_{n}$. Using (77) we can replace $\Delta \delta_{T}$ by

$$
L(a) \otimes 1+(\mathrm{id} \otimes L) \Delta(a),
$$


where $\Delta$ is the coassociative coproduct in $\mathcal{H}_{n}$. Thus we can use the notation which encodes the coassociativity of $\mathcal{H}_{n}$,

$$
\Delta a=a_{(1)} \otimes a_{(2)},(\mathrm{id} \otimes \Delta) \Delta(a)=(\Delta \otimes \mathrm{id}) \Delta(a)=a_{(1)} \otimes a_{(2)} \otimes a_{(3)} .
$$

The first term of 72 is then: $L(a) \otimes 1 \otimes 1+a_{(1)} \otimes \Delta \circ L a_{(2)}$, which by (77) gives

$$
L(a) \otimes 1 \otimes 1+a_{(1)} \otimes L\left(a_{(2)}\right) \otimes 1+a_{(1)} \otimes a_{(2)} \otimes L a_{(3)} .
$$

The second term of 72 is $\Delta \circ L(a) \otimes 1+\Delta a_{(1)} \otimes L a_{(2)}$, which by (77) gives,

$$
L(a) \otimes 1 \otimes 1+a_{(1)} \otimes L a_{(2)} \otimes 1+a_{(1)} \otimes a_{(2)} \otimes L a_{(3)} .
$$

Thus we conclude that $\Delta$ is coassociative.

We shall now characterize the Hopf algebra $\mathcal{H}_{R}=\cup \mathcal{H}_{n}$ as the solution of a universal problem in Hochschild cohomology. First, given an algebra $\mathcal{A}$ with augmentation $\varepsilon$, let us consider the Hochschild cohomology of $\mathcal{A}$ with coefficients in the following bimodule $\mathcal{M}$. As a vector space $\mathcal{M}=\mathcal{A}$, the left action of $\mathcal{A}$ on $\mathcal{M}$ is $(a, \xi) \rightarrow a \xi$, for all $a \in \mathcal{A}, \xi \in \mathcal{M}$. The right action of $\mathcal{A}$ on $\mathcal{M}$ is by $(\xi, a) \rightarrow \xi \varepsilon(a), \xi \in \mathcal{M}, a \in \mathcal{A}$. Thus the right module structure is through the augmentation. Let us denote the corresponding cocycles by $Z_{\varepsilon}^{n}(\mathcal{A})$, the coboundaries by $B_{\varepsilon}^{n}(\mathcal{A})$ and the cohomology as $H_{\varepsilon}^{n}(\mathcal{A})$.

Thus for instance a 1-cocycle $D \in Z_{\varepsilon}^{n}(\mathcal{A})$ is a linear map $\mathcal{A} \stackrel{D}{\rightarrow} \mathcal{A}$ such that $D(a b)=D(a) \varepsilon(b)+a D(b) \quad \forall a, b \in \mathcal{A}$. Next, given a Hopf algebra $\mathcal{H}$ we use the unit of $\mathcal{H}$ and its coalgebra structure to transpose (as in the Harrison cohomology), the above complex.

More precisely an $n$-cochain $L$ is a linear map,

$$
L: \mathcal{H} \rightarrow \underbrace{\mathcal{H} \otimes \ldots \otimes \mathcal{H}}_{n \text { times }}
$$

and the coboundary $b$ is given by,

$$
\begin{gathered}
(b L)(a)=(\mathrm{id} \otimes L) \Delta(a)-\Delta_{(1)} L(a)+\Delta_{(2)} L(a)+\ldots+(-1)^{j} \Delta_{(j)} L(a) \\
+\ldots+(-1)^{n} \Delta_{(n)} L(a)+(-1)^{n+1} L(a) \otimes 1
\end{gathered}
$$

where the lower index $(j)$ in $\Delta_{(j)}$ indicates where the coproduct is applied. For $n=0, L$ is just a linear form on $\mathcal{H}$ and one has

$$
(b L)(a)=(\mathrm{id} \otimes L) \Delta(a)-L(a) 1 .
$$

For $n=1, L$ is a linear map from $\mathcal{H}$ to $\mathcal{H}$ and

$$
(b L)(a)=(\mathrm{id} \otimes L) \Delta(a)-\Delta L(a)+L(a) \otimes 1 \in \mathcal{H} \otimes \mathcal{H} .
$$

We shall use the notation $Z_{\varepsilon}^{n}\left(\mathcal{H}^{*}\right), H_{\varepsilon}^{n}\left(\mathcal{H}^{*}\right) \ldots$ for the corresponding cocycles, cohomology classes, etc ... 
Theorem 2. There exists a pair $(\mathcal{H}, L)$, unique up to isomorphism, where $\mathcal{H}$ is a commutative Hopf algebra and $L \in Z_{\varepsilon}^{1}\left(\mathcal{H}^{*}\right)$ which is universal among all such pairs. In other words for any pair $\left(\mathcal{H}_{1}, L_{1}\right)$ where $\mathcal{H}_{1}$ is a commutative Hopf algebra and $L \in Z_{\varepsilon}^{1}\left(\mathcal{H}_{1}^{*}\right)$, there exists a unique Hopf algebra morphism $\mathcal{H} \stackrel{\rho}{\rightarrow} \mathcal{H}_{1}$ such that $L_{1} \circ \rho=\rho \circ L$.

Proof. Let $\mathcal{H}_{R}$ be the Hopf algebra of rooted trees and $L$ be the linear map defined by (73).

The equality (74) shows that $b L=0$. This shows that $L$ is a 1 -cocycle. It is clear that it is not a coboundary, indeed one has

$$
L(1)=\delta * \neq 0
$$

where $*$ is the tree with only one vertex.

Moreover, for any coboundary $T=b Z$ one has

$$
T(1)=0,
$$

since $T(1)=Z(1) 1-($ id $\otimes Z) \Delta(1)=0$.

Next consider a pair $\left(\mathcal{H}_{1}, L_{1}\right)$ where $\mathcal{H}_{1}$ is a commutative Hopf algebra and $L_{1} \in Z_{\varepsilon}^{1}\left(\mathcal{H}_{1}^{*}\right)$ is a 1-cocycle. The equality $L_{1} \circ \rho=\rho \circ L$ uniquely determines an algebra homomorphism $\rho: \mathcal{H}_{R} \rightarrow \mathcal{H}_{1}$. Indeed on the linear basis $\Pi \delta_{T_{i}}$ of $\mathcal{H}_{R}$ one must have,

$$
\rho\left(\Pi \delta_{T_{i}}\right)=\Pi \rho\left(\delta_{T_{i}}\right),
$$

by multiplicativity of $\rho$, while $\rho\left(\delta_{T}\right)$ is determined by induction by $\rho\left(\delta_{*}\right)=$ $L_{1}(1)$, and,

$$
\rho\left(L\left(\Pi \delta_{T_{i}}\right)\right)=L_{1} \rho\left(\Pi \delta_{T_{i}}\right) .
$$

We need to check that it is a morphism of Hopf algebras, i.e. that it is compatible with the coproduct,

$$
(\rho \otimes \rho)(\Delta(a))=\Delta_{1} \rho(a) \quad \forall a \in \mathcal{H}_{\mathcal{R}} .
$$

It is enough to check (91) on generators of the form $\delta_{T}=L\left(\Pi \delta_{T_{i}}\right)$. To do this one uses the cocycle property of $L_{1}$ which allows to write,

$$
\Delta_{1} L_{1}\left(\rho\left(\Pi \delta_{T_{i}}\right)\right)=L_{1}\left(\rho\left(\Pi \delta_{T_{i}}\right)\right) \otimes 1+\left(\mathrm{id} \otimes L_{1}\right) \Delta_{1} \rho\left(\Pi \delta_{T_{i}}\right) .
$$

One uses an induction hypothesis on the validity of (91), to write,

$$
\left(\mathrm{id} \otimes L_{1}\right) \Delta_{1} \rho\left(\Pi \delta_{T_{i}}\right)=(\rho \otimes(\rho \circ L)) \Delta\left(\Pi \delta_{T_{i}}\right)
$$

making use of the identity $\rho \otimes \rho \circ L=\left(\right.$ id $\left.\otimes L_{1}\right)(\rho \otimes \rho)$. Thus one has,

$$
\Delta_{1} L_{1}\left(\rho\left(\Pi \delta_{T_{i}}\right)\right)=L_{1}\left(\rho\left(\Pi \delta_{T_{i}}\right)\right) \otimes 1+(\rho \otimes(\rho \circ L)) \Delta\left(\Pi \delta_{T_{i}}\right),
$$

and the validity of (91) for $a=\delta_{T}$ follows from the equality $(\rho \otimes \rho) \Delta\left(L\left(\Pi \delta_{T_{i}}\right)\right)=$ $\left.(\rho \otimes \rho)\left(L\left(\Pi \delta_{T_{i}}\right)\right) \otimes 1+(\mathrm{id} \otimes L) \Delta\left(\Pi \delta_{T_{i}}\right)\right)$. 
We have thus shown the existence and uniqueness of the Hopf algebra morphism $\rho$.

As the simplest example, let $\mathcal{H}_{1}$ be the Hopf algebra of polynomials $P\left(\delta_{1}\right)$, as above, with,

$$
\Delta \delta_{1}=\delta_{1} \otimes 1+1 \otimes \delta_{1} .
$$

The cohomology group $H_{\varepsilon}^{1}\left(\mathcal{H}_{1}^{*}\right)$ is one dimensional, and the natural generator is the cocycle,

$$
L_{1}(P)(x)=\int_{0}^{x} P(a) d a \quad \forall P=P\left(\delta_{1}\right) \in \mathcal{H}_{1} .
$$

The cocycle identity follows from the equality,

$$
\int_{0}^{x+y} P(a) d a=\int_{0}^{x} P(a) d a+\int_{0}^{y} P(x+a) d a .
$$

The coboudaries are of the form,

$$
L_{0}(P)=\int(P(x+a)-P(a)) \phi(a) d a \quad \forall P=P\left(\delta_{1}\right) \in \mathcal{H}_{\infty},
$$

where $\phi$ is a distribution with support the origin, and possibly infinite order.

The tranpose $\rho^{t}$ of the morphism of Hopf algebras given by Theorem 2 determines a Lie algebra homomorphism from the one dimensional Lie algebra $\left(\mathcal{A}_{1}^{1}\right.$ with the notations of section $\left.\mathrm{I}\right)$, to the Lie algebra $\mathcal{L}^{1}$ which corresponds, by the Milnor-Moore theorem to the commutative Hopf algebra $\mathcal{H}_{R}$.

We proceed as in section I to determine $\mathcal{L}^{1}$.

Let $\mathcal{L}^{\infty}$ be the linear span of the elements $Z_{T}$, indexed by rooted trees. We introduce an operation on $\mathcal{L}^{1}$ by the equality,

$$
Z_{T_{1}} * Z_{T_{2}}=\sum_{T} n\left(T_{1}, T_{2} ; T\right) Z_{T}
$$

where the integer $n\left(T_{1}, T_{2} ; T\right)$ is determined as the number of simple cuts $c$ of cardinality 1 such that the cut branch is $T_{1}$ while the remaining trunk is $T_{2} \cdot{ }^{2}$

Theorem 3. a) Let $\mathcal{L}^{1}$ be the linear span of the elements $Z_{T}$, indexed by rooted trees. The following equality defines a structure of Lie algebra on $\mathcal{L}^{1}$. The Lie bracket $\left[Z_{T_{1}}, Z_{T_{2}}\right]$ is $Z_{T_{1}} * Z_{T_{2}}-Z_{T_{2}} * Z_{T_{1}}$.

b) The Hopf algebra $\mathcal{H}_{R}$ is the dual of the envelopping algebra of the Lie algebra $\mathcal{L}^{1}$.

Define

$$
A\left(T_{1}, T_{2}, T_{3}\right)=Z_{T_{1}} *\left(Z_{T_{2}} * Z_{T_{3}}\right)-\left(Z_{T_{1}} * Z_{T_{2}}\right) * Z_{T_{3}} .
$$

\footnotetext{
${ }^{2}$ The reader shall not confuse the operation which relates $T_{1}$ and $T_{2}$ to $T$ with the transplantation used in the theory of operads. Indeed, in the latter, the root of the tree $T_{1}$ is restricted to be the end of a branch of $T_{2}$.
} 
We shall need the following lemma,

Lemma 4. One has $A\left(T_{1}, T_{2}, T_{3}\right)=\Sigma n\left(T_{1}, T_{2}, T_{3} ; T\right) Z_{T}$, where the integer $n$ is the number of simple cuts $c$ of $T,|c|=2$ such that the two branches are $T_{1}, T_{2}$ while $R_{c}(T)=T_{3}$.

Proof. When one evaluates (100) against $Z_{T}$ one gets the coefficient,

$$
\sum_{T^{\prime}} n\left(T_{1}, T^{\prime} ; T\right) n\left(T_{2}, T_{3} ; T^{\prime}\right)-\sum_{T^{\prime \prime}} n\left(T_{1}, T_{2} ; T^{\prime \prime}\right) n\left(T^{\prime \prime}, T_{3} ; T\right),
$$

the first sum corresponds to pairs of cuts, $c, c^{\prime}$ of $T$ with $|c|=\left|c^{\prime}\right|=1$ and where $c^{\prime}$ is a cut of $R_{c}(T)$. These pairs of cuts fall in two classes, either $c \cup c^{\prime}$ is a simple cut or it is not. The second sum corresponds to pairs of cuts $c_{1}, c_{1}^{\prime}$ of $T$ such that $\left|c_{1}\right|=\left|c_{1}^{\prime}\right|=1, R_{c_{1}}(T)=T_{3}$ and $c_{1}^{\prime}$ is a cut of $P_{c_{1}}(T)$. In such a case $c_{1} \cup c_{1}^{\prime}$ is never a simple cut so the difference (101) amounts to substract from the first sum the pairs $c, c^{\prime}$ such that $c \cup c^{\prime}$ is not a simple cut. This gives,

$$
A\left(T_{1}, T_{2}, T_{3}\right)=\sum_{T} n\left(T_{1}, T_{2}, T_{3} ; T\right) Z_{T},
$$

where $n\left(T_{1}, T_{2}, T_{3} ; T\right)$ is the number of simple cuts $c$ of $T$ of cardinality 2 such that the two cut branches are $T_{1}$ and $T_{2}$.

It is thus clear that,

$$
A\left(T_{1}, T_{2}, T_{3}\right)=A\left(T_{2}, T_{1}, T_{3}\right) .
$$

One then computes $\left[\left[Z_{T_{1}}, Z_{T_{2}}\right], Z_{T_{3}}\right]+\left[\left[Z_{T_{2}}, Z_{T_{3}}\right], Z_{T_{1}}\right]+\left[\left[Z_{T_{3}}, Z_{T_{1}}\right], Z_{T_{2}}\right]$.

One can write it, for short, as a sum of 24 terms,

$$
\begin{aligned}
& \left(T_{1} * T_{2}\right) * T_{3}-\left(T_{2} * T_{1}\right) * T_{3}-T_{3} *\left(T_{1} * T_{2}\right)+T_{3} *\left(T_{2} * T_{1}\right) \\
+ & \left(T_{2} * T_{3}\right) * T_{1}-\left(T_{3} * T_{2}\right) * T_{1}-T_{1} *\left(T_{2} * T_{3}\right)+T_{1} *\left(T_{3} * T_{2}\right) \\
+ & \left(T_{3} * T_{1}\right) * T_{2}-\left(T_{1} * T_{3}\right) * T_{2}-T_{2} *\left(T_{3} * T_{1}\right)+T_{2} *\left(T_{1} * T_{3}\right) \\
= & -A\left(T_{1}, T_{2}, T_{3}\right)+A\left(T_{2}, T_{1}, T_{3}\right)-A\left(T_{3}, T_{1}, T_{2}\right)+A\left(T_{3}, T_{2}, T_{1}\right) \\
& -A\left(T_{2}, T_{3}, T_{1}\right)+A\left(T_{1}, T_{3}, T_{2}\right)=0 .
\end{aligned}
$$

b) For each rooted tree $T$ let us define a linear form $Z_{T}$ on $\mathcal{H}_{R}$ by the equality,

$$
\left\langle Z_{T}, P\left(\delta_{T_{i}}\right)\right\rangle=\left(\partial / \partial \delta_{T} P\right)(0) .
$$

Thus $Z_{T}$ vanishes when paired with any monomial $\delta_{T_{1}}^{n_{1}} \ldots \delta_{T_{k}}^{n_{k}}$ except when this monomial is $\delta_{T}$ while,

$$
\left\langle Z_{T}, \delta_{T}\right\rangle=1 \text {. }
$$

Since $P \rightarrow P(0)$ is the counit of $\mathcal{H}_{R}$ and since $Z_{T}$ satisfies

$$
\left\langle Z_{T}, P Q\right\rangle=\left\langle Z_{T}, P\right\rangle \varepsilon(Q)+\varepsilon(P)\left\langle Z_{T}, Q\right\rangle,
$$


it follows that the coproduct of $Z_{T}$ is,

$$
\Delta Z_{T}=Z_{T} \otimes 1+1 \otimes Z_{T},
$$

where the coproduct on $\mathcal{H}_{R}^{*}$ is defined, when it makes sense, by dualizing the product of $\mathcal{H}_{R}$.

Similarly the product of two elements of $\mathcal{H}_{R}^{*}$ is defined by

$$
\left\langle Z_{1} Z_{2}, P\right\rangle=\left\langle Z_{1} \otimes Z_{2}, \Delta P\right\rangle .
$$

Since the bracket of two derivations is still a derivation, the subspace of $\mathcal{H}_{R}^{*}$ of elements satisfying (102) is stable under bracket. What remains is to show that,

$$
Z_{T_{1}} Z_{T_{2}}-Z_{T_{2}} Z_{T_{1}}=\left[Z_{T_{1}}, Z_{T_{2}}\right],
$$

where the r.h.s. is defined by the Lie algebra structure of theorem 3.

Let $\mathcal{H}_{0}$ be the augmentation ideal of $\mathcal{H}_{R}, \mathcal{H}_{0}=\operatorname{Ker} \varepsilon$. The formula defining the coproduct in $\mathcal{H}_{R}$ shows that,

$$
\Delta \delta_{T}=\delta_{T} \otimes 1+1 \otimes \delta_{T}+R_{T}
$$

where $R_{T} \in \mathcal{H}_{0} \otimes \mathcal{H}_{0}$. In fact one can compute $R_{T}$ modulo higher powers of $\mathcal{H}_{0}$, i.e. modulo $\mathcal{H}_{0}^{2} \otimes \mathcal{H}_{0}$, it gives,

$$
R_{T}^{(0)}=\sum_{c} \delta_{T_{c}^{\prime}} \otimes \delta_{T_{c}}
$$

where $c$ varies among single cuts of the tree $T$, where $T_{c}$ is the part of $T$ that contains the base point, while $T_{c}^{\prime}$ is the tree which remains. When one computes $\left\langle Z_{T_{1}} Z_{T_{2}}, \Pi \delta_{T_{i}}\right\rangle=\left\langle Z_{T_{1}} \otimes Z_{T_{2}}, \Pi \Delta \delta_{T_{i}}\right\rangle$ the part which is not symmetric in $T_{1}, T_{2}$ is zero unless $\Pi \delta_{T_{i}}$ is equal to a single $\delta_{T}$. When one computes

$$
\left\langle Z_{T_{1}} Z_{T_{2}}, \delta_{T}\right\rangle=\left\langle Z_{T_{1}} \otimes Z_{T_{2}}, \Delta \delta_{T}\right\rangle,
$$

the only part which contributes comes from $R_{T}^{(0)}$ and it counts the number of ways of obtaining $T$ from $T_{1}$ and $T_{2}$, which gives (109).

Proposition 5. The equality degree $Z_{T}=\#$ of vertices of $T$ defines a grading of the Lie algebra $\mathcal{L}^{1}$.

Proof. The number of vertices of any tree obtained by gluing $T_{1}$ to $T_{2}$ is the sum of the number of vertices of $T_{1}$ and $T_{2}$.

We shall now show how to extend the Hopf algebra $\mathcal{H}_{R}$ to include the generators $X, Y$ of the Lie algebra of the affine group as in section I. The commutator of $Y$ with $\delta_{T}$ will simply be given by,

$$
\left[Y, \delta_{T}\right]=\operatorname{deg}(T) \delta_{T}
$$


i.e. by the above grading.

The commutator with $X$ will generate a derivation $N$ of $\mathcal{H}_{R}$, uniquely determined by its value on the generators $\delta_{T}$, by

$$
N \delta_{T}=\sum \delta_{T^{\prime}}
$$

where the trees $T^{\prime}$ are obtained by adding one vertex and one edge to $T$ in all possible ways without changing the base point. It is clear that the sum (114) contains $\operatorname{deg}(T)$ terms.

Using the derivation property of $N$, one has,

$$
N\left(\prod_{1}^{n} \delta_{T_{i}}\right)=\sum_{1}^{n} \delta_{T_{1}} \ldots N\left(\delta_{T_{i}}\right) \ldots \delta_{T_{n}} .
$$

Our first task will be to get a formula for $\Delta N\left(\delta_{T}\right)$.

Proposition 6. For any $a \in \mathcal{H}_{\mathcal{R}}$ one has

$$
\Delta N a=(N \otimes \text { id }) \Delta a+(\text { id } \otimes N) \Delta a+\left[\delta_{1} \otimes Y, \Delta a\right] .
$$

Proof. First, it is enough to check the equality when $a=\delta_{T}$. Indeed, both $\Delta \circ N$ and $\left(N \otimes \mathrm{id}+\mathrm{id} \otimes N+\operatorname{ad}\left(\delta_{1} \otimes Y\right)\right) \circ \Delta$ are derivations from $\mathcal{H}_{R}$ to the $\mathcal{H}_{R}$-bimodule $\mathcal{H}_{R} \otimes \mathcal{H}_{R}$ (using $\Delta$ to define the bimodule structure). Thus so is their difference $\varepsilon_{0}$ which vanishes provided it does on the generators $\delta_{T}$. Let thus $T$ be a pointed tree and $T^{\prime}$ be obtained from $T$ by adjoining an edge at $v_{0} \in \Delta^{0}(T)$. One has

$$
\Delta \delta_{T^{\prime}}=\delta_{T^{\prime}} \otimes 1+1 \otimes \delta_{T^{\prime}}+\sum_{c^{\prime}}\left(\Pi \delta_{T_{j}^{\prime}} \otimes \delta_{R_{c^{\prime}}^{\prime}}\right)
$$

where $c^{\prime} \in \Delta^{1}\left(T^{\prime}\right)$ varies among the simple cuts of $T^{\prime}$. One has $\Delta^{1}\left(T^{\prime}\right)=$ $\Delta^{1}(T) \cup\{\varepsilon\}$ where $\varepsilon$ the new edge. Now the cuts $c^{\prime}$ for $T^{\prime}$ are of two kinds,

(A) The new edge is not cut, (A') It is cut.

There is also another dichotomy,

(B) The vertex $v_{0}$ belongs to the trunk, (B') It belongs to one of the cut branches.

If we sum (116) over all possible $T^{\prime}$ we get,

$$
\Delta N \delta_{T}=N \delta_{T} \otimes 1+1 \otimes N \delta_{T}+\sum_{v} \sum_{c^{\prime}} \Pi \delta_{T_{i}^{\prime}} \otimes \delta_{R_{c^{\prime}}} .
$$

Let us concentrate on the last term and consider first only the cuts $c^{\prime}$ which satisfy (A) We also consider the term,

$$
\sum_{c}\left(\Pi \delta_{T_{c}}\right) \otimes \delta_{R_{c}}
$$


over all the cuts $c$ of the tree $T$. If we apply (id $\otimes N)$ to (118), we obtain all possible cuts of a $T^{\prime}$ such that (A) (B) holds so that,

$$
\sum_{(A)(B)}=(\mathrm{id} \otimes N) \sum_{c}\left(\Pi \delta_{T_{c}}\right) \otimes \delta_{R_{c}} .
$$

It follows that,

$$
\sum_{(A)\left(B^{\prime}\right)}=(N \otimes \mathrm{id}) \sum_{c}\left(\Pi \delta_{T_{c}}\right) \otimes \delta_{R_{c}} .
$$

We can thus summarize what we obtained so far by,

$$
\Delta N \delta_{T}=(N \otimes \mathrm{id}) \Delta \delta_{T}+(\mathrm{id} \otimes N) \Delta \delta_{T}+\sum_{\left(A^{\prime}\right)} .
$$

Now consider the sum $\sum_{\left(A^{\prime}\right)}$, the first case is when the only cut is the cut of the new edge. The only cut branch gives us a $\delta_{1}$ and the number of ways of doing it is $n=\operatorname{deg} T$, thus we get

$$
\left[\delta_{1} \otimes Y, \delta_{T} \otimes 1+1 \otimes \delta_{T}\right],\left[Y, \delta_{T}\right]=n \delta_{T} .
$$

The next case is when a non trivial cut $c$ remains after we remove the new edge. For that cut $c$ the new vertex necessarily belongs to the trunk (so that (A) (B) is excluded) as follows from the very definition of a cut. For such cuts, the result is to get an additional $\delta_{1}$ among the $\delta_{T_{i}}$, which comes from the cut new edge. The number of ways of doing it is exactly the degree of the trunk. Thus we get

$$
\left[\delta_{1} \otimes Y, \sum_{c}\left(\Pi \delta_{T_{c}}\right) \otimes \delta_{R_{c}}\right] .
$$

Combining (122) and (123) we get,

$$
\sum_{\left(A^{\prime}\right)}=\left[\delta_{1} \otimes Y, \Delta \delta_{T}\right]
$$

This is enough to assert that for any tree $T$ one has,

$$
\Delta N \delta_{T}=(N \otimes \mathrm{id}) \Delta \delta_{T}+(\mathrm{id} \otimes N) \Delta \delta_{T}+\left[\delta_{1} \otimes Y, \Delta \delta_{T}\right]
$$

which ends the proof of Proposition 6.

In other words we can enlarge $\mathcal{H}_{R}$ to $\widetilde{\mathcal{H}}_{R}$ by adjoining the elements $X, Y$ with

$$
\begin{array}{cc}
{[X, a]} & =N(a),[Y, a]=(\operatorname{deg} a) a \quad \forall a \in \mathcal{H}_{R} \\
{[Y, X]=X, \Delta Y} & =Y \otimes 1+1 \otimes Y, \Delta X=X \otimes 1+1 \otimes X+\delta_{1} \otimes Y .
\end{array}
$$


Let us translate Proposition 6 in terms of the transposed map $N^{t}$ acting on $\mathcal{H}_{R}^{*}$.

One has $\left\langle N^{t}(A B), a\right\rangle=\langle A B, N(a)\rangle=$

$$
\begin{gathered}
\langle A \otimes B, \Delta N(a)\rangle=\left\langle A \otimes B,\left(N \otimes \mathrm{id}+\mathrm{id} \otimes N+\delta_{1} \otimes \operatorname{deg}\right) \Delta a\right\rangle \\
=\left\langle N^{t}(A) \otimes B+A \otimes N^{t}(B)+\left(\delta_{1}\right)^{t} \otimes \operatorname{deg}^{t}(A \otimes B), \Delta a\right\rangle \\
=\left\langle N^{t}(A) B+A N^{t}(B)+\delta_{1}^{t}(A) \operatorname{deg}^{t}(B), a\right\rangle ;
\end{gathered}
$$

thus,

$$
N^{t}(A B)=N^{t}(A) B+A N^{t}(B)+\delta_{1}^{t}(A) \operatorname{deg}^{t}(B),
$$

where $\delta_{1}^{t}\left(\right.$ resp. $\left.\operatorname{deg}^{t}\right)$ is the transposed of the multiplication by $\delta_{1}$ (resp. deg)

$$
\left\langle\delta_{1}^{t} A, a\right\rangle=\left\langle A, \delta_{1} a\right\rangle .
$$

One has $\left\langle\delta_{1}^{t}(A B), a\right\rangle=\left\langle A B, \delta_{1} a\right\rangle=\left\langle A \otimes B, \Delta \delta_{1} \Delta a\right\rangle=\left\langle A \otimes B,\left(\delta_{1} \otimes 1+1 \otimes\right.\right.$ $\left.\left.\delta_{1}\right) \Delta a\right\rangle$. Thus,

$$
\delta_{1}^{t}(A B)=\delta_{1}^{t}(A) B+A \delta_{1}^{t}(B),
$$

i.e. $\delta_{1}^{t}$ is a derivation. Moreover on the generator $Z_{T}$,

$$
\delta_{1}^{t}\left(Z_{T}\right)=0 \quad \text { unless } \quad T=\{*\}, \delta_{1}^{t}\left(Z_{1}\right)=1 .
$$

Indeed, $\left\langle Z_{T}, \delta_{1} a\right\rangle=0$ unless $T=\{*\}$, while for $T=\{*\}$ one gets that $\left\langle Z_{1}, \delta_{1} a\right\rangle=\varepsilon(a)$. Thus,

$$
\delta_{1}^{t}=\frac{\partial}{\partial Z_{1}}
$$

where we use the Poincaré-Birkhoff-Witt theorem to write elements of $\mathcal{U}\left(\mathcal{L}^{1}\right)$ in the form $\sum \Pi Z_{T_{i}} Z_{1}^{a}$.

Let us compute $N^{t}\left(Z_{T}\right)$ where $T$ is a tree with more than one vertex. One has $\left\langle N^{t} Z_{T}, \delta_{T_{1}} \delta_{T_{2}} \ldots \delta_{T_{n}}\right\rangle=\left\langle Z_{T}, N\left(\delta_{T_{1}} \ldots \delta_{T_{n}}\right)\right\rangle$, and this vanishes unless $n=1$. Moreover for $n=1$,

$$
\left\langle Z_{T}, N\left(\delta_{T_{1}}\right)\right\rangle=n\left(T ; T_{1}\right)
$$

where $n\left(T ; T_{1}\right)$ is the number of times the tree $T$ is obtained by adjoining an edge and vertex to $T_{1}$.

Thus one has,

$$
N^{t} Z_{T}=\sum n\left(T ; T_{1}\right) Z_{T_{1}} \quad, N^{t} Z_{1}=0 .
$$

We can now state the analogue of Lemma 4 of Section I as follows, where we let $\mathcal{L}^{k}$ be the Lie subalgebra of $\mathcal{L}^{1}$ generated by the $Z_{T}$ with $\operatorname{deg}(T) \geq k$.

Lemma 7. When restricted to $\mathcal{U}\left(\mathcal{L}^{2}\right), N^{t}$ is the unique derivation, with values in $\mathcal{U}\left(\mathcal{L}^{1}\right)$ satisfying (133), moreover, for $\operatorname{deg}\left(T_{i}\right)>1$ and $A=\Pi Z_{T_{i}}$ one has

$$
N^{t}\left(A Z_{1}^{a_{1}}\right)=N^{t}(A) Z_{1}^{a_{1}}+A \frac{a_{1}\left(a_{1}-1\right)}{2} Z_{1}^{a_{1}-1} .
$$


Proof. The first statement follows from $(127)$ and $(129)$. The second statement follows from,

$$
N^{t}\left(Z_{1}^{m}\right)=\frac{m(m-1)}{2} Z_{1}^{m-1}
$$

which one proves by induction on $m$.

Motivated by Section I and the first part of the lemma, we enlarge the Lie algebra $\mathcal{L}^{1}$ by adjoining two elements $Z_{0}$ and $Z_{-1}$ such that,

$$
\begin{gathered}
{\left[Z_{-1}, Z_{1}\right]=Z_{0},\left[Z_{0}, Z_{T}\right]=\operatorname{deg}(T) Z_{T}} \\
{\left[Z_{-1}, Z_{T}\right]=\sum n\left(T ; T_{1}\right) Z_{T_{1}} \quad \forall T, \operatorname{deg}(T)>1 .}
\end{gathered}
$$

The obtained Lie algebra $\mathcal{L}$, is an extension of the Lie algebra of formal vector fields with $Z_{0}=x \frac{\partial}{\partial x}, Z_{-1}=\frac{\partial}{\partial x}$ and as above $Z_{n}=\frac{x^{n+1}}{(n+1) !} \frac{\partial}{\partial x}$, as follows from,

Theorem 8. The following equality defines a surjective Lie algebra homomorphism from $\mathcal{L}$ to $\mathcal{A}$,

$$
\Theta\left(Z_{T}\right)=n(T) Z_{n}, \quad \Theta\left(Z_{i}\right)=Z_{i}, i=0,1
$$

where $n(T)$ is the number of times $\delta_{T}$ occurs in $N^{\operatorname{deg}(T)-1}\left(\delta_{1}\right)$.

Proof. The elements $X, Y$, and $\delta_{*}$ of the Hopf algebra $\widetilde{\mathcal{H}}_{R}$ fulfill the presentation of section I for the Hopf algebra $\widetilde{\mathcal{H}}_{T}$, thus there exists a unique homorphism of Hopf algebras $h$ from $\widetilde{\mathcal{H}}_{R}$ to $\widetilde{\mathcal{H}}_{T}$ such that,

$$
h(X)=X, \quad h(Y)=Y, \quad h\left(\delta_{1}\right)=\delta_{*} .
$$

By construction, $h$ restricts to the subalgebra $\mathcal{H}_{R}$ and defines a homomorphism to the Hopf algebra $\mathcal{H}_{T}$. Transposing this homomorphism to the Lie algebras, one obtains the restriction of $\Theta$ to the subalgebra $\mathcal{L}^{1}$.

At this stage we completed our understanding of the relation between the two Hopf algebras. It is best expressed by the Lie algebra homomorphism $\Theta$ from $\mathcal{L}^{1}$ to $\mathcal{A}^{1}$. Its extension to the full $\mathcal{L}$ justifies the construction of the latter Lie algebra.

By Theorem 3 the Hopf algebra $\mathcal{H}_{R}$ should be thought of as the algebra of coordinates on a nilpotent formal group $\mathcal{G}$ whose Lie algebra is the graded Lie algebra $\mathcal{L}^{1}$. Given a field $K$, elements of the group $\mathcal{G}_{K}$ are obtained precisely as the characters of the algebra $\mathcal{H}_{R} \otimes_{\mathbf{Q}} K$. Indeed, such characters correspond to group-like elements $u$ (i.e. elements $u$ satisfying $\Delta(u)=u \otimes u$ ) of a suitable completion of the envelopping algebra of $\mathcal{L}^{1}$. Viewing $u$ as a linear form on $\mathcal{H}_{R}$ gives us the desired character. If we let $K$ be the field of formal power series in a variable $\epsilon$ we thus obtain as points of $\mathcal{G}_{K}$ the homomorphisms from $\mathcal{H}_{R}$ to $K$. 
It is not difficult to check that the map which to every bare Feynman diagram $\Gamma$ associates the corresponding Laurent expansion (in Dimensional Regularization, say, with regularized dimension $D=4-2 \epsilon$, in four dimensions, say) is precisely such a character.

This allows to reduce by the above conceptual mathematical structure of inversion in $\mathcal{G}$ the computation of renormalization in QFT to the primitive elements of the Hopf algebra,i.e. to Feynman diagrams without subdivergences.

In order to better understand the extension of the group of diffeomorphisms provided by the group $\mathcal{G}$, it would be desirable to find a non-commutative manifold $X$, whose diffeomorphism group is $\mathcal{G}$.

The coordinates $\delta_{n}=-\left(\log \left(\psi^{\prime}(x)\right)^{(n)}\right.$ of a diffeomorphism $\psi$ allow to reconstruct the latter by the formula

$$
\psi(x)=\int_{0}^{x} \exp \left(-\sum \frac{\delta_{n}}{n !} u^{n}\right) d u .
$$

This formula provides the clear meaning both for composition and inversion of diffeomorphisms.

Of course, we would love to have a similar formula for the group $\mathcal{G}$ and it is tantalizing to consider the Feynman integral

$$
\int \exp \left(-L_{0}+\sum_{\Gamma} L_{\Gamma}\right)
$$

as a direct analogue of the above expression.

\section{Acknowledgements}

D.K. thanks the I.H.E.S., Bures-sur-Yvette, for hospitality during a stay Jan.Feb. 1998 and the theory group at the CPT (Marseille) for interest and discussions. Also, support by a Heisenberg Fellowship of the DFG for D.K. is gratefully acknowledged. D.K. thanks R. Stora for motivating the investigation which will be reported in [7].

\section{References}

[1] D. Kreimer, On the Hopf Algebra Structure of Perturbative Quantum Field Theories, Adv.Theor.Math.Phys.2.2 (1998) 303-334; q-alg/9707029.

[2] A. Connes, H. Moscovici, Hopf Algebras, Cyclic Cohomology and the Transverse Index Theorem, IHES publication IHES/M/98/37 (1998).

[3] J. Dixmier, Existence de traces non normales, C.R.Acad.Sci.Paris, Ser.AB262 (1966), A1107-A1108.

[4] J.C. Collins, Renormalization, Cambridge Univ.Press (1984). 
[5] D. Kreimer, Renormalization and Knot Theory, J.Knot Th.Ram.6 (1997) 479-581, a-alg/9607022.

[6] D.J. Broadhurst, R. Delbourgo, D. Kreimer, Unknotting the polarized vacuum of quenched QED, Phys.Lett.B366 (1996) 421, hep-ph/9509296.

[7] D. Kreimer, On Overlapping Divergences, in preparation.

[8] T. Krajewski, R. Wulkenhaar, On Kreimer's Hopf Algebra Structure of Feynman Graphs, CPT-98/P.3639; hep-th/9805098.

\section{Appendix}

\section{1 $\quad \phi^{3}$ Theory and Overlapping Divergences}

A prominent problem in renormalization theory is the presence of overlapping divergences. We will soon see that to Green functions which suffer from such overlapping divergences we will have to associate a sum of trees, while so far our experience only lead to the identification of single trees with a given Green function.

We will proceed by studying the example of $\phi^{3}$ theory in six dimensions. A full study will be given elsewhere [7], but we also mention that solutions to the problem of overlapping divergences were already found in [5], using combinatorical considerations concerning divergent sectors, in [1] and [6] using Schwinger Dyson equations, and were also known to others. In $[7]$ we will show how overlapping divergences give rise to a slightly modified Hopf algebra, which eventually turns out to be identical to the Hopf algebra of rooted trees considered here. We sketch this more formal argument after the consideration of $\phi^{3}$ theory as an example.

In whatever approach one takes, the final message is the same: Overlapping divergent functions can be resolved in sums of functions having only nested and disjoint divergences. To see how this comes about, we will here employ yet another approach, using differential equations on bare Green functions.

Green functions in $\phi_{6}^{3}$ theory which are overall divergent are provided by two- and three-point functions, to which we refer as $G_{n}^{[2]}(q ; m)$ and $G_{n}^{[3]}(p, q ; m)$. Here the subscript $n$ refers to the number of loops in the Green-function, and $m$ is the mass of the propagator, while $p, q$ are external momenta.

We first consider $G_{n}^{[3]}(p, q ; m)$ :

$$
G_{n}^{[3]}(p, q ; m)=\int d^{6} l_{1} \ldots d^{6} l_{n} \prod_{i=1}^{3 n} \frac{1}{P_{i}} .
$$

For $n \geq 1$, it is a product of $3 n$ propagators $P_{i}=1 /\left(k_{i}^{2}-m^{2}+i \eta\right)$, where the $k_{i}$ are momentum vectors which are linear combinations of external momenta 
$p, q$ and $n$ internal momenta $l_{1}, \ldots, l_{n}$ such that momentum conservation holds at each vertex.

As each propagator $1 / P_{i}$ contributes with weight two to the powercounting, we find that $G^{[3]}$ is overall logarithmic divergent, $3 \times n \times 2-6 \times n=0$.

For each $P_{i}$, let $\overline{P_{i}}:=k_{i}^{2}+i \eta$, so that $P_{i}=\overline{P_{i}}-m^{2}$.

Then, one immediately sees that $G_{n}^{[3]}(p, q ; m)-G_{n}^{[3]}(p, q ; 0)$ is overall convergent. This follows directly from powercounting in the expression

$$
\prod_{i:=1}^{3 n} \frac{\prod_{j} \overline{P_{j}}-\prod_{j}\left(\overline{P_{j}}-m^{2}\right)}{P_{i} \overline{P_{i}}}
$$

Thus, to determine the counterterm for a vertex function, it suffices to consider the massless case. ${ }^{3}$

Hence all possible subdivergences of $G_{n}^{[3]}(p, q ; 0)$ are given by functions of the type $G_{r}^{[3]}\left(k_{i}, k_{j} ; 0\right)$ and $G_{s}^{[2]}\left(k_{i} ; 0\right)$, with $s<n$ and $r<n$.

In the context of $\phi^{3}$ theory in six dimensions, overlapping divergences can only be provided by two-point functions. The only circumstance which stops us to assign a unique tree to $G_{n}^{[3]}(p, q ; m)$ is the fact that there might be overlapping subdivergences provided by massless two-point functions $G_{s}^{[2]}\left(k_{i} ; 0\right), s<n$.

Before we handle these subdivergences, we turn to $G_{n}^{[2]}(q ; m)$ itself. At $n$ loops, it consists of $3 n-1$ propagators

$$
G_{n}^{[2]}(q ; m)=\int d^{6} l_{1} \ldots d^{6} l_{n} \prod_{i:=1}^{3 n-1} \frac{1}{P_{i}}
$$

Consider the difference

$$
G_{n}^{[2]}(q ; m)-G_{n}^{[2]}(q ; 0)=m^{2} \int d^{6} l_{1} \ldots d^{6} l_{n} \prod_{i:=1}^{3 n-1} \frac{1}{P_{i}} \sum_{j:=1}^{3 n-1} \frac{1}{\overline{P_{j}}}
$$

+overall finite terms

which is of overall logarithmic degree of divergence. As far as the overall counterterm is concerned, we can even nullify masses in this difference and thus find that the divergences of $G_{n}^{[2]}(q ; m)$ can be separated as

$$
\begin{aligned}
G_{n}^{[2]}(q ; m)= & m^{2} \int d^{6} l_{1} \ldots d^{6} l_{n} \prod_{i:=1}^{3 n-1} \frac{1}{\overline{P_{i}}} \sum_{j:=1}^{3 n-1} \frac{1}{\overline{P_{j}}} \\
& +G_{n}^{[2]}(q ; 0)+U(q, m)
\end{aligned}
$$

where $U(q ; m)$ collects all the overall finite terms.

\footnotetext{
${ }^{3}$ Even better, again using powercounting, one immediately shows that it is sufficient to consider $G_{n}^{[3]}(0, q ; 0)$.
} 
The first term on the rhs is overall logarithmic divergent. It only can provide overlapping divergences through massless functions $G_{s}^{[2]}(q ; 0)$ appearing as subgraphs in it, quite similar to the analysis of the vertex function, as the sum over $j$ squares one propagator in turn.

We have thus reduced all appearances of overlapping divergences to the presence of functions $G_{i}^{[2]}(q ; 0), i \leq n$. It remains to show how the overlapping divergences in $G_{n}^{[2]}(q ; 0)$ can be handled for all $n$.

This is actually not that difficult. Necessarily, $G_{n}^{[2]}(q ; 0)$ has the form

$$
G_{n}^{[2]}(q ; 0)=\left(q^{2}\right)^{1-n \epsilon} F_{G_{n}}(\epsilon)
$$

where $F_{G_{n}}(\epsilon)$ is a Laurent series in $\epsilon$. Hence $G_{n}^{[2]}(q ; 0)$ fulfils the differential equation

$$
\frac{1}{2 D(1-n \epsilon)} q^{2} \frac{\partial}{\partial q_{\mu}} \frac{\partial}{\partial q^{\mu}} G_{n}^{[2]}(q ; 0)=G_{n}^{[2]}(q ; 0) .
$$

This solves the problem. The remaining source of overlapping divergences, $G_{n}^{[2]}(q ; 0)$, is expressed in terms of the overall logarithmic divergent function $q^{2} \frac{\partial}{\partial q_{\mu}} \frac{\partial}{\partial q^{\mu}} G_{n}^{[2]}(q ; 0)$ which is free of overlapping divergences. Such an approach is also very useful in practice [6].

Fig.(14) gives two examples for the resolution of overlapping divergences. Crosses in the figure indicate where the derivatives with respect to $q$ act for a chosen momentum flow through the graph.

\section{A general Argument}

So far, we decomposed graphs which have overlapping divergences into a sum of contributions each of which delivers a rooted tree. Thus, overlapping divergences correspond to a linear combination of rooted trees, while any Feynman diagram without overlapping divergences corresponds to a single rooted tree.

One might suggest to enlargen the Hopf algebra $H_{R}$ of rooted trees to another Hopf algebra, $H_{O}$ say, so that $H_{O}$ directly contains elements which correspond to graphs with overlapping divergences [8].

Let us at this stage mention a general fact which shows that any such Hopf algebra $H_{O}$ is nothing else than the Hopf algebra of rooted trees. If we take into account the decorations of vertices by Feynman diagrams without subdivergences, any such Hopf algebra $H_{O}$ is a Hopf algebra $H_{R}$ for an appropriate set of decorations.

Consider a Feynman graph $\Gamma$ which has overlapping subdivergences, but in a way that any of its divergent subgraphs $\gamma \subset \Gamma$ and any of the complementary graphs $\Gamma / \gamma$ is free of overlapping subdivergences. The first example in Fig.(14) is of this type. The cases we have excluded here will be handled later by a recursive argument. 


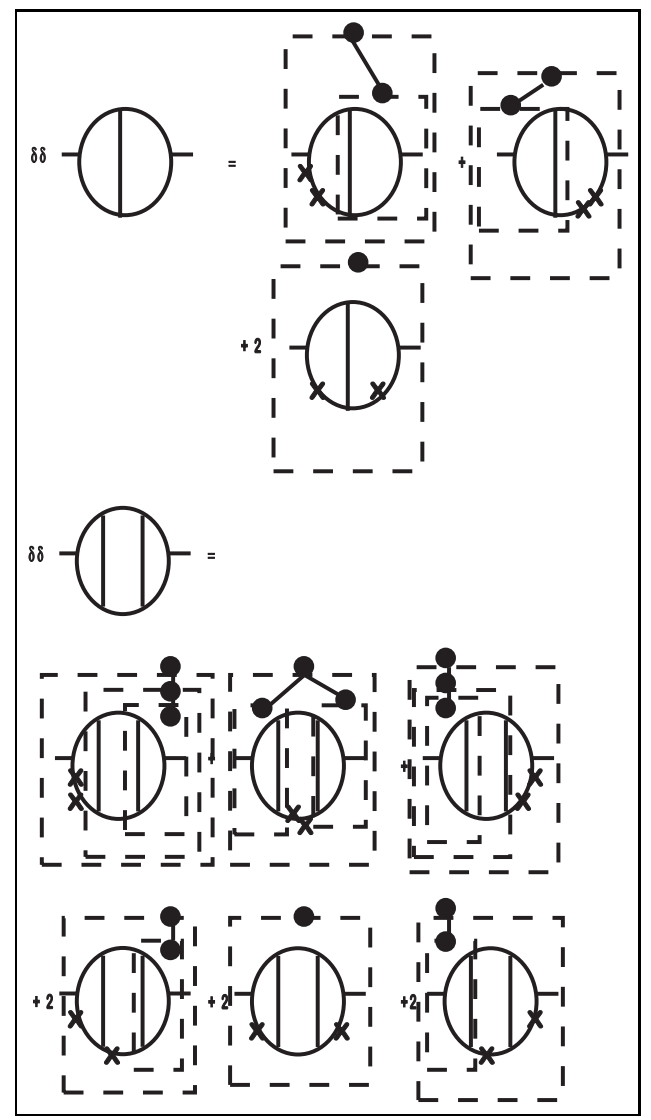

Figure 14: The resolution of overlapping divergences and the resulting sum of trees. A double derivative with respect to the external momentum resolves the graph in contributions each of which is free of overlapping divergences. We indicate by crosses on propagators the places where the derivative acts, for a chosen momentum flow. 
We want to construct a Hopf algebra $H_{O}$ which contains a single element $t_{\Gamma}$ such that the antipode $S\left(t_{\Gamma}\right)$ delivers the counterterm without making recourse to the methods of the previous paragraph to disentangle $t_{\Gamma}$ first as a sum of trees $t_{i}$ in some decorated algebra $H_{R}$. The question is: Could such an algebra have a structure different from $H_{R}$ ?

Now, as $H_{O}$ shall also be able to treat Feynman diagrams which only have nested or disjoint subdivergences, it will contain the Hopf algebra of rooted trees as a subalgebra.

Let us actually construct $H_{O}$ by fairly general arguments. Let $H_{R} \subset H_{O}$ be given, and let in particular all Feynman graphs without subdivergences be identified. Hence all possible decorations, and thus all primitive elements of $H_{R}$ are assumed to be determined. Note that the primitive elements of $H_{O}$ are identical with the primitive elements of $H_{R}$ as graphs with overlapping divergences necessarily contain subdivergences, and thus do not provide primitive elements per se.

A Feynman graph $\Gamma$ chosen as above has only subgraphs which can be described by proper rooted trees. Thus, its coproduct in $H_{O}$ will have the general form

$$
\Delta\left(t_{\Gamma}\right)=t_{\Gamma} \otimes e+e \otimes t_{\Gamma}+\sum_{\gamma} t(\gamma) \otimes t(\Gamma / \gamma)
$$

where the sum is over all subgraphs of $\Gamma$, while $t(\gamma)$ and $t(\Gamma / \gamma)$ are the rooted trees assigned to the corresponding graphs. By the constraints which we imposed on $\Gamma$ this is always possible. In $H_{O}$ we consider the above equation as the definition for the coproduct on elements $t_{\Gamma} \notin H_{R} \subset H_{O}$. is

On the rhs of the above coproduct, the only part which is not in $H_{R} \otimes H_{R}$

$$
t_{\Gamma} \otimes e+e \otimes t_{\Gamma}
$$

and we write

$$
\Delta\left(t_{\Gamma}\right)=t_{\Gamma} \otimes e+e \otimes t_{\Gamma}+R_{\Gamma}
$$

with $R_{\Gamma} \in H_{R} \otimes H_{R}$.

Now, we know that there exists an element $T_{\Gamma} \in H_{R}$ such that

$$
\Delta\left(T_{\Gamma}\right)=T_{\Gamma} \otimes e+e \otimes T_{\Gamma}+R_{\Gamma}
$$

This element $T$ is just the linear combination of rooted trees constructed in the previous section, but ist existences can be established on general grounds from the consideration of maximal forests [5, 7].

Finally we set $U:=t_{\Gamma}-T_{\Gamma}$ and calculate

$$
\Delta(U)=U \otimes e+e \otimes U
$$

Now, if $U$ is superficially divergent at all it is a primitive element. It thus can be described by the rooted tree $t_{1}$. To be able to do so we only have to enlarge 
the algebra $H_{R}$ to contain the decoration $U$. An easy recursion argument finally allows to drop the constraint on $\Gamma$ [7].

One concludes that any Hopf algebra which contains $H_{R}$ but also elements $t_{\Gamma} \notin H_{R}$ is isomorphic to the algebra of rooted trees $H_{R}$ with an enlarged set of primitive elements. In Fig.(14) we see some contributions which only generate the tree $t_{1}$. They correspond to such new primitive elements. A detailed version of this argument will be given elsewhere [7]. 